University of Wollongong

Research Online

Faculty of Engineering - Papers (Archive)

Faculty of Engineering and Information

Sciences

August 1993

\title{
Theory of the piezo-Zeeman effect for shallow acceptors in group-IV semiconductors
}

\author{
K. J. Duff \\ University of Wollongong
}

Follow this and additional works at: https://ro.uow.edu.au/engpapers

Part of the Engineering Commons

https://ro.uow.edu.au/engpapers/269

\section{Recommended Citation}

Duff, K. J.: Theory of the piezo-Zeeman effect for shallow acceptors in group-IV semiconductors 1993. https://ro.uow.edu.au/engpapers/269

Research Online is the open access institutional repository for the University of Wollongong. For further information contact the UOW Library: research-pubs@uow.edu.au 


\title{
Theory of the piezo-Zeeman effect for shallow acceptors in group-IV semiconductors
}

\author{
K. J. Duff \\ Department of Physics, University of Wollongong, Northfields Avenue, Wollongong, New South Wales 2522, Australia
}

(Received 30 November 1992)

Theory is presented for the energy splittings and transition intensities for a single hole system initially of $T_{d}$ symmetry which is then subject to the simultaneous application of a uniaxial stress and uniform magnetic field. Four combinations of stress and field directions are considered.

\section{INTRODUCTION}

It is axiomatic that semiconductors are of immense technological significance, and that the scope of such materials derives from the introduction into the lattice of controlled levels of impurities. It is therefore of considerable continuing experimental interest ${ }^{1}$ to characterize the states associated with the impurities, and one important technique for accomplishing this is optical spectroscopy. The discriminating factors used in the interpretation of spectra are line splittings, intensity variations of spectral components when perturbations of suitable symmetries are applied, and intensity comparisons between polarizations of the radiation. It is important that theory be available relating the splittings and intensities to the parameters under experimental control. The simultaneous application of a magnetic field and uniaxial stress is a particularly powerful interpretive instrument, ${ }^{2}$ and so it is the purpose of this work to provide theory appropriate to an impurity subject to both perturbations. It is assumed that the impurity, in the absence of the perturbations, occupies a site of $T_{d}$ symmetry as befits a substitutional impurity in the elemental semiconductors.

The literature to date carries two distinct but complementary streams of theoretical support for the experimental studies of impurity spectra. The first relies on a numerical/variational solution of the effective-mass equations to generate energy eigenvalues for ground and excited states. This procedure has been carried out with increasing technical sophistication. Satisfactory results have been obtained for a range of impurities in the unperturbed crystal, ${ }^{3}$ for the crystal subject to uniaxial stress, ${ }^{4}$ for the crystal subject to a magnetic field, ${ }^{5,6}$ and for hydrostatic pressure. ${ }^{6}$ Because the method yields wave functions, transition amplitudes can be calculated, so in some cases transition intensities were obtained. No calculations of this kind have been reported for the combined application of uniaxial stress and magnetic field. The dominant advantage of this methodology is its proximity to first principles.

The alternate procedure utilizes the principles of group theory to predict relative splittings and relative intensities for the various transitions and polarizations that are possible. The inputs to the theory are simply the assumed site symmetry of the impurity under study, and the symmetry of the applied perturbations including that of the dipole moment operator appropriate to the radiation field. Because this theory is not a "first-principles" theory in the sense of the methodologies mentioned in the previous paragraph, its predictions are given in terms of a few material-dependent parameters which may be obtained from the experimental data or from calculations of the aforementioned kind. The strengths of this approach are its portability from one system to another (subject to the input of the appropriate set of material-dependent parameters), and its intrinsic inclusion within one formalism of nonlinear effects arising from interactions under perturbation among members of what in zero perturbation constitutes a single manifold of states. The procedure can be extended ${ }^{7}$ to include interactions between members of different manifolds, but this is not routinely attempted. Application of such group-theoretical procedures have been made to impurity states in strained ${ }^{8,9}$ semiconductors and also to the Zeeman effect. ${ }^{10,11}$

The methodology presented here for the piezo-Zeeman effect follows the second of the two schema. Each Hamiltonian is simply the sum of the Hamiltonians used for stress in Ref. 8 and for the Zeeman effect in Ref. 10. The stress Hamiltonians are linear in displacements, but the Zeeman Hamiltonians have both linear and quadratic terms. Four combinations of stress and field directions have been chosen, in all four cases the stress and field being orthogonal to each other. In each case the combined Hamiltonian is diagonalized exactly. The unitary transformations thereby generated are then used in the standard manner to construct the transition amplitudes, and intensities calculated.

Some of the results derived here have already been reported, ${ }^{12}$ but without description of the procedure. Subsequent to these reports, other work has been reported by Villaret and Rodriguez ${ }^{13}$ in which similar methodology has been used with some overlap in application with the present work; however, they have not included terms quadratic in field in their Zeeman Hamiltonian. Those authors present results for stress and field parallel, and this has not been attempted in the present paper, but where they report results for stress and field orthogonal, they do so for only one stress direction, whereas three directions are reported here. Also, they have not given consideration to an intensity parameter which is here shown to be needed under certain observation conditions. Thus, although there is some overlap, the two works are largely complementary. 


\section{THEORY}

The states of interest here are classified according to the double-valued representations of the group $\bar{T}_{d}$, namely $\Gamma_{6}, \Gamma_{7}$, and $\Gamma_{8}$, these being two-, two-, and fourdimensional representations respectively. In the presence of both uniaxial stress and uniform magnetic field, the Hamiltonian is simply the sum of the separate Zeeman and stress Hamiltonians. Bhattercharjee and Rodriguez ${ }^{10}$ have constructed the Zeeman Hamiltonians to second order in magnetic field $\mathbf{B}$, and these are

$$
\begin{aligned}
& H_{Z}^{(6)}=\mu_{B} g^{(6)} \mathbf{B} \cdot \mathrm{j}+q^{(6)} B^{2}, \\
& H_{Z}^{(7)}=\mu_{B} g^{(7)} \mathbf{B} \cdot \mathrm{j}+q^{(7)} B^{2},
\end{aligned}
$$

and

$$
\begin{aligned}
H_{Z}^{(8)}= & \mu_{B} g_{1}^{\prime} \mathbf{B} \cdot \mathbf{J}+\mu_{B} g_{2}^{\prime}\left(B_{x} J_{x}^{3}+B_{y} J_{y}^{3}+B_{z} J_{z}^{3}\right)+q_{1} B^{2} \\
& +q_{2}(\mathbf{B} \cdot \mathbf{J})^{2}+q_{3}\left(B_{x}^{2} J_{x}^{2}+B_{y}^{2} J_{y}^{2}+B_{z}^{2} J_{z}^{2}\right)
\end{aligned}
$$

In these equations $\mu_{B}$ is the Bohr magneton; the components of $\mathbf{j}$ are $j_{x}, j_{y}$, and $j_{z}$, the $2 \times 2$ angular momentum matrices for $j=\frac{1}{2}$; the components of $\mathbf{J}$ are $J_{x}, J_{y}$, and $J_{z}$, the $4 \times 4$ angular momentum matrices for $J=\frac{3}{2}$; $g^{(6)}, g^{(7)}, g_{1}^{\prime}, g_{2}^{\prime}, q^{(6)}, q^{(7)}, q_{1}, q_{2}$, and $q_{3}$ are coupling parameters. The explicit forms of the angular momentum matrices depend on the choices of phases for the basis functions for the representations; the choices made in Ref. 10 are used here and are listed in the Appendix. It will frequently be convenient to treat perturbations to $\Gamma_{6}$ and $\Gamma_{7}$ states together, in which case a superscript $(6,7)$ will be used to indicate a choice may be made.

The piezo-Hamiltonian for the $\Gamma_{8}$ states has been constructed by Rodriguez, Fisher, and Barra ${ }^{8}$ using as basis states functions that are compatible with those used for the Zeeman Hamiltonian above. Their expression is

$$
\begin{aligned}
H_{p}^{(8)}= & a^{\prime} I\left(\varepsilon_{x x}+\varepsilon_{y y}+\varepsilon_{z z}\right)+b^{\prime}\left[\varepsilon_{x x}\left(J_{x}^{2}-\frac{5}{4} I\right)+\varepsilon_{y y}\left(J_{y}^{2}-\frac{5}{4} I\right)+\varepsilon_{z z}\left(J_{z}^{2}-\frac{5}{4} I\right)\right] \\
& +\left(2 d^{\prime} / \sqrt{3}\right)\left[\left\{J_{y} J_{z}\right\} \varepsilon_{y z}+\left\{J_{z} J_{x}\right\} \varepsilon_{z x}+\left\{J_{x} J_{y}\right\} \varepsilon_{x y}\right] .
\end{aligned}
$$

Here $\left\{J_{i} J_{j}\right\}$ is $\frac{1}{2}\left(J_{i} J_{j}+J_{j} J_{i}\right) ; a^{\prime}, b^{\prime}$, and $d^{\prime}$ are the (phenomenological) deformation-potential constants; $I$ is the $4 \times 4$ unit matrix; the srain tensor $\varepsilon_{i j}$ is defined as

$$
\varepsilon_{i j}=\frac{1}{2}\left[\frac{\partial u_{i}}{\partial x_{j}}+\frac{\partial u_{j}}{\partial x_{i}}\right] \quad(i, j=x, y, z),
$$

where $\mathbf{u}(\mathbf{r})$ is the displacement induced by the stress. Kramers' theorem requires that there be no splitting of a $\Gamma_{6}$ or $\Gamma_{7}$ level under the application of stress, so the piezo-Hamiltonian for these states is simply a multiple of the $2 \times 2$ unit matrix, viz.

$$
H_{p}^{(6,7)}=a^{(6,7)} I\left(\varepsilon_{x x}+\varepsilon_{y y}+\varepsilon_{z z}\right) .
$$

For general orientations of the stress and magneticfield axes, the theory is too cumbersome for convenient study, so in this work a few directions of high symmetry have been chosen. The theory uses the same methodolo- gy for each of these selected combinations of field and stress directions. In each case, the first step in the diagonalization of the Hamiltonian is to transform to a set of states quantized with respect to the magnetic-field direction by the application of suitable rotation operators. After this, the problem is no more difficult than the diagonalization of $2 \times 2$ matrices. For the $\Gamma_{6}$ and $\Gamma_{7}$ states, Kramers' theorem requires that the piezo-Hamiltonian be a multiple of the $2 \times 2$ unit matrix, so the construction of the functions just mentioned is all that is required.

Relative intensities for optical transitions are calculated for the possible transitions, namely those derived from the unperturbed transitions $\Gamma_{8} \rightarrow \Gamma_{6}, \quad \Gamma_{8} \rightarrow \Gamma_{7}$, and $\Gamma_{8} \rightarrow \Gamma_{8}$. The starting point for each such calculation is the relevant transition amplitude matrix as constructed by previous workers ${ }^{8,10}$ for transitions between unperturbed states. These amplitudes are given in Eqs. (68), (70), and (71) of Ref. 10.

$$
\begin{aligned}
& \underline{Q}^{(8 \rightarrow 6)}=D_{0}\left[\begin{array}{cccc}
\sqrt{3}(\hat{\mathbf{x}}+i \hat{\mathbf{y}}) & -2 i \widehat{\mathbf{z}} & (\hat{\mathbf{x}}-i \hat{\mathbf{y}}) & 0 \\
0 & i(\hat{\mathbf{x}}+i \widehat{\mathbf{y}}) & 2 \widehat{\mathbf{z}} & i \sqrt{3}(\hat{\mathbf{x}}-i \hat{\mathbf{y}})
\end{array}\right], \\
& \underline{Q}^{(8 \rightarrow 7)}=D_{0}^{\prime}\left(\begin{array}{cccc}
-(\hat{\mathbf{x}}-i \hat{\mathbf{y}}) & 0 & \sqrt{3}(\hat{\mathbf{x}}+i \hat{\mathbf{y}}) & 2 i \widehat{\mathbf{z}} \\
-2 \widehat{\mathbf{z}} & i \sqrt{3}(\widehat{\mathbf{x}}-i \hat{\mathbf{y}}) & 0 & -i(\hat{\mathbf{x}}+i \hat{\mathbf{y}})
\end{array}\right), \\
& \underline{Q}^{(8 \rightarrow 8)}=\left(\begin{array}{cccc}
0 & -\left(D+2 D^{\prime}\right)(\widehat{\mathbf{x}}+i \widehat{\mathbf{y}}) & -i\left(D-D^{\prime}\right) \hat{\mathbf{z}} & \sqrt{3} D^{\prime}(\hat{\mathbf{x}}-i \widehat{\mathbf{y}}) \\
-D(\widehat{\mathbf{x}}-i \widehat{\mathbf{y}}) & 0 & \sqrt{3} D^{\prime}(\widehat{\mathbf{x}}+i \widehat{\mathbf{y}}) & -i\left(D+3 D^{\prime}\right) \widehat{\mathbf{z}} \\
i\left(D+3 D^{\prime}\right) \widehat{\mathbf{z}} & -\sqrt{3} D^{\prime}(\widehat{\mathbf{x}}-i \widehat{\mathbf{y}}) & 0 & D(\widehat{\mathbf{x}}+i \widehat{\mathbf{y}}) \\
-\sqrt{3} D^{\prime}(\widehat{\mathbf{x}}+i \widehat{\mathbf{y}}) & i\left(D-D^{\prime}\right) \widehat{\mathbf{z}} & \left(D+2 D^{\prime}\right)(\widehat{\mathbf{x}}-i \widehat{\mathbf{y}}) & 0
\end{array}\right) .
\end{aligned}
$$


In these matrices $D_{0}, D_{0}^{\prime}, D$, and $D^{\prime}$ are complex amplitude parameters. If $\widehat{\mathbf{q}}$ is the unit vector in the direction of polarization of the radiation, the amplitude for any transition is found by taking the scalar product of $\hat{\mathbf{q}}$ with the indicated matrix element; $\hat{\mathbf{q}}$ may describe circular polarization if it has an imaginary component, for example, for radiation propagating in the direction $\widehat{\mathbf{z}}$, left circular polarization has $\hat{\mathbf{q}}_{L}=(\hat{\mathbf{x}}+i \widehat{\mathbf{y}}) / \sqrt{2}$, and right circular polarization is described by $\hat{\mathbf{q}}_{R}=(\hat{\mathbf{x}}-i \hat{\mathbf{y}}) / \sqrt{2}$. The intensity (strictly speaking, the transition probability) is, as usual, the square modulus of the amplitude. In the tables of intensities for $\Gamma_{8} \rightarrow \Gamma_{6}$ and $\Gamma_{8} \rightarrow \Gamma_{7}$ transitions, only relative intensities of the various components are listed; the overall normalization factor for the former is $2\left|D_{0}\right|^{2}$ and for the latter it is $2\left|D_{0}^{\prime}\right|^{2}$. For the $\Gamma_{8} \rightarrow \Gamma_{8}$ transitions, the normalization factor is implicit in the definitions of the intensity parameters used in the tables.

\section{A. Case 1: stress parallel to [100], magnetic field parallel to [001]}

\section{Energies and wave functions}

The strain components for this orientation are $\varepsilon_{x x}=s_{11} T, \varepsilon_{y y}=\varepsilon_{z z}=s_{12} T$, and $\varepsilon_{x y}=\varepsilon_{y z}=\varepsilon_{z x}=0$, where $T$ is the stress and $s_{11}$ and $s_{12}$ are the usual compliance coefficients. The only crystal symmetry elements that are common to both the applied stress and the applied magnetic field are $E, \bar{E}, C_{2}$, and $\bar{C}_{2}$, the latter being rotations through $\pi$ about the magnetic-field direction. Thus the eigenfunctions belong to the group $\bar{C}_{2}$. The character table for this group is listed in Table I. All representations are one dimensional, that is, all degeneracies are lifted. The Hamiltonians are

$$
\begin{aligned}
& H^{(6,7)}=\mu_{B} g^{(6,7)} B j_{z}+q^{(6,7)} B^{2} I+a^{(6,7)}\left(s_{11}+2 s_{12}\right) T I \\
& H_{Z}^{(8)}=\mu_{B} B\left(g_{1}^{\prime} J_{z}+g_{2}^{\prime} J_{z}^{3}\right)+B^{2}\left(q_{1}+q_{2} J_{z}^{2}+q_{3} J_{z}^{2}\right) \\
& H_{p}^{(8)}=a^{\prime}\left(s_{11}+2 s_{12}\right) T I+b^{\prime}\left(s_{11}-s_{12}\right) T\left(J_{x}^{2}-\frac{5}{4} I\right)
\end{aligned}
$$

Clearly $H^{(6,7)}$ are diagonal, so the $\Gamma_{6}\left(\bar{T}_{d}\right)$ and $\Gamma_{7}\left(\bar{T}_{d}\right)$ basis functions are the eigenfunctions in the presence of the perturbations. The energy eigenvalues are

$$
E_{ \pm 1 / 2}^{(6,7)}=q^{(6,7)} B^{2}+a^{(6,7)}\left(s_{11}+2 s_{12}\right) T \pm \frac{1}{2} \mu_{B} g^{(6,7)} B
$$

By considering a rotation through $\pi$ about the $z$ axis, the basis functions $\psi_{1 / 2}^{(6,7)}$ are seen to belong to $\Gamma_{3}\left(\bar{C}_{2}\right)$, and $\psi_{-1 / 2}^{(6,7)}$ belong to $\Gamma_{4}\left(\bar{C}_{2}\right)$.

Because the operators of the group $\bar{C}_{2}$ are a subset of the operators of the group $\bar{T}_{d}$, the functions $\psi_{\mu}^{(8)}\left(\bar{T}_{d}\right)$ used for the construction of the interaction Hamiltonians of Eqs. (11) and (12) are eigenfunctions of each of the symmetry operators of the group $\bar{C}_{2}$. Because the functions $\psi_{\mu}^{(8)}$ generate the angular momentum matrices, they satisfy

$$
J_{z} \psi_{\mu}^{(8)}=\mu \psi_{\mu}^{(8)}
$$

and so, for the $C_{2}$ operation, we have

$$
e^{i \pi J_{z}} \psi_{\mu}^{(8)}=e^{i \mu \pi} \psi_{\mu}^{(8)}
$$

Thus the character for this operation is $e^{i \mu \pi}$, which for $\mu=\frac{3}{2}$ and $-\frac{1}{2}$ is $-i$ so $\psi_{3 / 2}^{(8)}$ and $\psi_{-1 / 2}^{(8)}$ belong to $\Gamma_{4}\left(\bar{C}_{2}\right)$; for $\mu=\frac{1}{2}$ and $-\frac{3}{2}$ the character is $i$, so $\psi_{1 / 2}^{(8)}$ and $\psi_{-3 / 2}^{(8)}$ belong to $\Gamma_{3}\left(\bar{C}_{2}\right)$. The Hamiltonian can only connect states of like symmetry, so it necessarily has the structure of two $2 \times 2$ on-diagonal matrices; however, with the rows and columns corresponding to $\mu$ in descending sequence, the $2 \times 2$ matrices are interleaved as follows:

$H^{(8)}=h_{0} I+\left(\begin{array}{cccc}h_{1}-h_{2} & 0 & h_{3} & 0 \\ 0 & h_{2}+h_{4} & 0 & h_{3}^{\prime} \\ h_{3}^{*} & 0 & h_{2}-h_{4} & 0 \\ 0 & h_{3}^{\prime *} & 0 & -h_{1}-h_{2}\end{array}\right)$,

where

$$
\begin{aligned}
& h_{0}=a^{\prime}\left(s_{11}+2 s_{12}\right) T+B^{2}\left[q_{1}+\frac{5}{4}\left(q_{2}+q_{3}\right)\right], \\
& h_{1}=\mu_{B} B\left(\frac{3}{2} g_{1}^{\prime}+\frac{27}{8} g_{2}^{\prime}\right), \\
& h_{2}=\frac{1}{2} b^{\prime}\left(s_{11}-s_{12}\right) T-\left(q_{2}+q_{3}\right) B^{2}, \\
& h_{3}=h_{3}^{\prime}=\frac{\sqrt{3}}{2} b^{\prime}\left(s_{12}-s_{11}\right) T, \\
& h_{4}=\mu_{B} B\left(\frac{1}{2} g_{1}^{\prime}+\frac{1}{8} g_{2}^{\prime}\right) .
\end{aligned}
$$

\begin{tabular}{|c|c|c|c|c|c|c|}
\hline $\begin{array}{l}\bar{C}_{2} \\
\bar{C}_{s} \\
\end{array}$ & $\begin{array}{l}E \\
E\end{array}$ & $\begin{array}{l}\bar{E} \\
\bar{E}\end{array}$ & $\begin{array}{c}C_{2} \\
\sigma \\
\end{array}$ & $\begin{array}{c}\bar{C}_{2} \\
\bar{\sigma} \\
\end{array}$ & $\begin{array}{l}\text { Bases for } \bar{C}_{2} \\
\text { (case } 1 \text { or } 2 \text { ) }\end{array}$ & $\begin{array}{l}\text { Bases for } \bar{C}_{2} \\
\text { (case } 3 \text { or } 4 \text { ) }\end{array}$ \\
\hline$\Gamma_{1}$ & 1 & 1 & 1 & 1 & $J_{z}$ or $z$ & $J_{z}$ or $x$ or $y$ \\
\hline$\Gamma_{2}$ & 1 & 1 & -1 & -1 & $J_{x}$ or $J_{y}$ or $x$ or $y$ & $J_{x}$ or $J_{y}$ or $z$ \\
\hline$\Gamma_{3}$ & 1 & -1 & $i$ & $-i$ & $\psi_{1 / 2}^{(6,7)}$ & $\psi_{+1 / 2}^{6}$ or $\psi_{-1 / 2}^{7}$ \\
\hline$\Gamma_{4}$ & 1 & -1 & $-i$ & $i$ & $\psi_{-1 / 2}^{(6,7)}$ & $\psi_{-1 / 2}^{6}$ or $\psi_{+1 / 2}^{7}$ \\
\hline
\end{tabular}

In Eq. (16) the matrix has been displayed in a form which is slightly more general than is here necessary, in order that it also have validity for other cases to be considered. Here $h_{3}$ is real. The unitary transformation that diagonalizes $H^{(8)}$ is

TABLE I. Character table for the groups $\bar{C}_{2}$ and $\bar{C}_{s}$. 
$S=\left(\begin{array}{cccc}\cos \theta & 0 & -\sin \theta e^{i \alpha} & 0 \\ 0 & \cos \theta^{\prime} & 0 & -\sin \theta^{\prime} e^{i \alpha^{\prime}} \\ \sin \theta e^{-i \alpha} & 0 & \cos \theta & 0 \\ 0 & \sin \theta^{\prime} e^{-i \alpha^{\prime}} & 0 & \cos \theta^{\prime}\end{array}\right)$

where

$$
\begin{aligned}
& e^{i \alpha}=h_{3} /\left|h_{3}\right|, \quad e^{i \alpha^{\prime}}=h_{3}^{\prime} /\left|h_{3}^{\prime}\right|, \\
& \sin 2 \theta=\frac{2\left|h_{3}\right|}{\sqrt{\left(h_{1}+h_{4}-2 h_{2}\right)^{2}+4\left|h_{3}\right|^{2}}}, \\
& \cos 2 \theta=\frac{h_{1}+h_{4}-2 h_{2}}{\sqrt{\left(h_{1}+h_{4}-2 h_{2}\right)^{2}+4\left|h_{3}\right|^{2}}}, \\
& \sin 2 \theta^{\prime}=\frac{2\left|h_{3}^{\prime}\right|}{\sqrt{\left(h_{1}+h_{4}+2 h_{2}\right)^{2}+4\left|h_{3}^{\prime}\right|^{2}}}, \\
& \cos 2 \theta^{\prime}=\frac{h_{1}+h_{4}+2 h_{2}}{\sqrt{\left(h_{1}+h_{4}+2 h_{2}\right)^{2}+4\left|h_{3}^{\prime}\right|^{2}}} .
\end{aligned}
$$

The eigenfunctions formed as linear combinations must have the same symmetry as the functions from which they are composed. Thus the functions specified by the columns of $S$ may be designated in sequence $\psi_{4}, \psi_{3}, \psi_{4^{\prime}}, \psi_{3^{\prime}}$, and the corresponding energy eigenvalues are

$E_{4}=h_{0}+\frac{1}{2}\left[h_{1}-h_{4}+\sqrt{\left(h_{1}+h_{4}-2 h_{2}\right)^{2}+4\left|h_{3}\right|^{2}}\right]$,

$E_{3}=h_{0}+\frac{1}{2}\left[h_{4}-h_{1}+\sqrt{\left(h_{1}+h_{4}+2 h_{2}\right)^{2}+4\left|h_{3}^{\prime}\right|^{2}}\right]$,

$E_{4^{\prime}}=h_{0}+\frac{1}{2}\left[h_{1}-h_{4}-\sqrt{\left(h_{1}+h_{4}-2 h_{2}\right)^{2}+4\left|h_{3}\right|^{2}}\right]$

$E_{3^{\prime}}=h_{0}+\frac{1}{2}\left[h_{4}-h_{1}-\sqrt{\left(h_{1}+h_{4}+2 h_{2}\right)^{2}+4\left|h_{3}^{\prime}\right|^{2}}\right]$.

\section{Intensities}

We consider in turn transitions to final states derived from the original $\Gamma_{6}, \Gamma_{7}$, and $\Gamma_{8}$ states, with the initial states constructed from a $\Gamma_{8}$ manifold. For the unperturbed transitions $\Gamma_{8} \rightarrow \Gamma_{6}$ the transition amplitude matrix is shown in Eq. (7). In the presence of the magnetic field and stress there is no mixing of the $\Gamma_{6}$ states, so the transition amplitudes are found by constructing $\underline{Q}^{(8 \rightarrow 6)} S$.

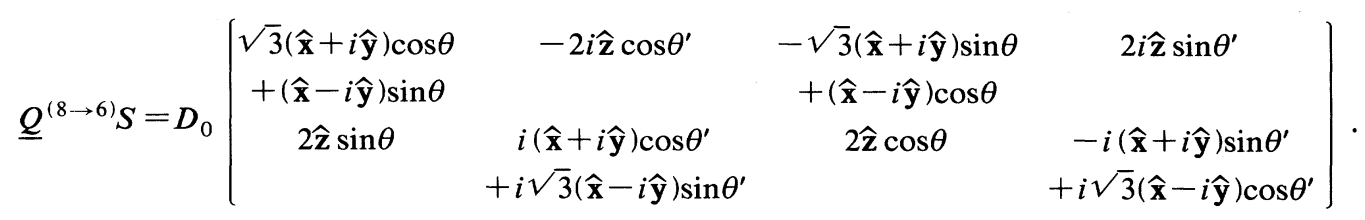

The intensities are found from these amplitudes in the standard manner. Some examples are listed in Table II.

The procedure is now repeated for the $\Gamma_{8} \rightarrow \Gamma_{7}$ transitions, this time starting with the amplitude matrix of Eq. (8). Once more there is no mixing of the final states in the presence of stress and field, so the transition amplitudes are obtained from $\underline{Q}^{(8 \rightarrow 7)} S$.

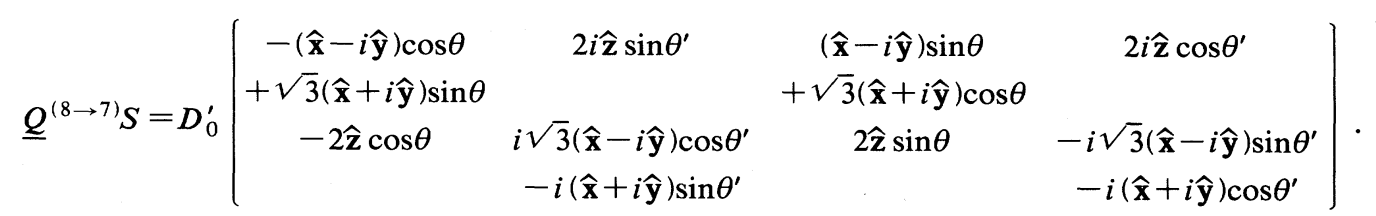

Intensities deduced from these amplitudes have been included in Table II.

For the $\Gamma_{8} \rightarrow \Gamma_{8}$ transitions, in the presence of the stress and the magnetic perturbations, both the initial and the final states have become mixtures of the unperturbed states, so the transition amplitudes are given by $S_{2}^{\dagger} \underline{Q}^{8 \rightarrow 8} S_{1}$, where $S_{1}$ and $S_{2}$ each have the structure displayed in Eq. (22). For convenience of notation the parameters $\theta$ and $\theta^{\prime}$ are retained for $S_{1}$, but are replaced by $\phi$ and $\phi^{\prime}$, respectively, for $S_{2}$. A compact representation of the result follows from the introduction of some convenient definitions, viz.

$$
\begin{aligned}
& f_{B}(\theta, \phi)=i\left[\left(D+3 D^{\prime}\right) \cos \theta \sin \phi-\left(D-D^{\prime}\right) \sin \theta \cos \phi\right] \\
& f_{T}(\theta, \phi)=D \sin \theta \sin \phi+\sqrt{3} D^{\prime}(\sin \theta \cos \phi-\cos \theta \sin \phi)-\left(D+2 D^{\prime}\right) \cos \theta \cos \phi \\
& \mathbf{F}(\hat{\mathbf{a}}, \hat{\mathbf{b}} ; \theta, \phi)=\widehat{\mathbf{a}} f_{T}(\theta, \phi)+\widehat{\mathbf{b}} f_{T}(-\theta,-\phi) .
\end{aligned}
$$

With these definitions, the amplitude matrix in the presence of both stress and magnetic field is 


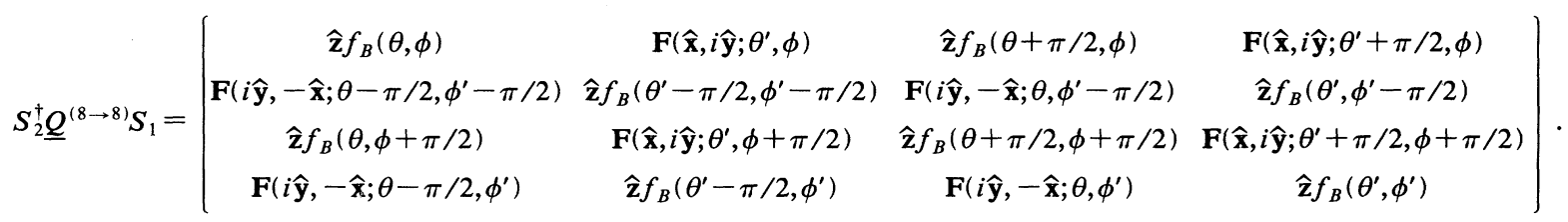

The intensities for these transitions are generally expressed ${ }^{8,10}$ in terms of a normalization $N$ and two real parameters $u$ and $v$ which may be defined in terms of the complex amplitude parameters through the relationships

$$
\begin{aligned}
& \left|D+D^{\prime}\right|^{2}=\frac{1}{4} N(1-u), \\
& \left|D-D^{\prime}\right|^{2}=\frac{1}{4} N(1+2 v), \\
& \left|D+3 D^{\prime}\right|^{2}=\frac{1}{4} N(1-2 v) .
\end{aligned}
$$

For polarization parallel to the magnetic field, the intensities are conveniently tabulated by expressing $\left|f_{B}\right|^{2}$ in terms of $N, u$, and $v$. For radiation polarized parallel to stress, $\left|f_{T}\right|^{2}$ is needed in terms of the same variables.

$$
\begin{aligned}
& \left|f_{B}(\theta, \phi)\right|^{2}=\frac{N}{8}\{1-\cos (2 \theta-2 \phi)+2 u \sin 2 \theta \sin 2 \phi+2 v[\cos 2 \phi-\cos 2 \theta]\} \\
& \left|f_{T}(\theta, \phi)\right|^{2}=\frac{N}{8}\left\{1+\cos (2 \theta+2 \phi)+2 u \sin \left(2 \theta+\frac{\pi}{3}\right) \sin \left[2 \phi-\frac{\pi}{3}\right]-2 v\left[\cos \left[2 \theta+\frac{\pi}{3}\right]+\cos \left[2 \phi-\frac{\pi}{3}\right]\right]\right\} .
\end{aligned}
$$

TABLE II. Relative intensities for $\Gamma_{8} \rightarrow \Gamma_{6}$ and $\Gamma_{8} \rightarrow \Gamma_{7}$ transitions. Angles $2 \theta$ and $2 \theta^{\prime}$ are defined in terms of field, stress, and coupling parameters in Eqs. (24) and (25), respectively. Radiation with circular polarization is assumed to be propagating parallel to the magnetic field. All entries are valid for the case of $\mathbf{B} \|[001]$ with $\mathbf{T} \|[100]$. The entries for linear polarization parallel to the magnetic field, and for circular polarization also apply to the case of $\mathbf{B} \|[001]$ with $\mathbf{T} \|[110]$, provided that the application of Eqs. (24) and (25) for $2 \theta$ and $2 \theta^{\prime}$ uses the set of parameters (84)-(88).

\begin{tabular}{cccccc}
\hline \hline $\bar{T}_{d}$ & $\bar{C}_{2}$ & $\Gamma_{4}$ & $\Gamma_{3}$ & $\Gamma_{4^{\prime}}$ & $\Gamma_{3^{\prime}}$ \\
\hline
\end{tabular}

$\begin{array}{lll}\Gamma_{6}\left(\frac{1}{2}\right) & \Gamma_{3} & 1+\cos (2 \theta-\pi / 3) \\ \Gamma_{6}\left(-\frac{1}{2}\right) & \Gamma_{4} & 0 \\ \Gamma_{7}\left(\frac{1}{2}\right) & \Gamma_{3} & 1-\cos (2 \theta-\pi / 3) \\ \Gamma_{7}\left(-\frac{1}{2}\right) & \Gamma_{4} & 0\end{array}$

(a) Radiation polarized parallel to stress

$$
\begin{array}{lll}
1-\cos \left(2 \theta^{\prime}+\pi / 3\right) & 0 & 1+\cos \left(2 \theta^{\prime}+\pi / 3\right) \\
0 & 1+\cos (2 \theta-\pi / 3) & 0 \\
1+\cos \left(2 \theta^{\prime}+\pi / 3\right) & 0 & 1-\cos \left(2 \theta^{\prime}+\pi / 3\right)
\end{array}
$$

$\begin{array}{lll}\Gamma_{6}\left(\frac{1}{2}\right) & \Gamma_{3} & 0 \\ \Gamma_{6}\left(-\frac{1}{2}\right) & \Gamma_{4} & 1-\cos 2 \theta \\ \Gamma_{7}\left(\frac{1}{2}\right) & \Gamma_{3} & 0 \\ \Gamma_{7}\left(-\frac{1}{2}\right) & \Gamma_{4} & 1+\cos 2 \theta\end{array}$

$\Gamma_{6}\left(\frac{1}{2}\right) \quad \Gamma_{3} \quad(1-\cos 2 \theta) / 2$

$\Gamma_{6}\left(-\frac{1}{2}\right) \quad \Gamma_{4} \quad 0$

$\Gamma_{7}\left(\frac{1}{2}\right) \quad \Gamma_{3} \quad(1+\cos 2 \theta) / 2$

$\begin{array}{lll}\Gamma_{7}\left(-\frac{1}{2}\right) & \Gamma_{4} & 0\end{array}$

$\begin{array}{lll}\Gamma_{6}\left(\frac{1}{2}\right) & \Gamma_{3} & 3(1+\cos 2 \theta) / 2 \\ \Gamma_{6}\left(-\frac{1}{2}\right) & \Gamma_{4} & 0 \\ \Gamma_{7}\left(\frac{1}{2}\right) & \Gamma_{3} & 3(1-\cos 2 \theta) / 2 \\ \Gamma_{7}\left(-\frac{1}{2}\right) & \Gamma_{4} & 0\end{array}$

(b) Radiation polarized parallel to magnetic field

$\begin{array}{lll}1+\cos 2 \theta^{\prime} & 0 & 1-\cos 2 \theta^{\prime} \\ 0 & 1+\cos 2 \theta & 0 \\ 1-\cos 2 \theta^{\prime} & 0 & 1+\cos 2 \theta^{\prime} \\ 0 & 1-\cos 2 \theta & 0\end{array}$

(c) Radiation with left circular polarization

$\begin{array}{lll}0 & (1+\cos 2 \theta) / 2 & 0 \\ 3\left(1-\cos 2 \theta^{\prime}\right) / 2 & 0 & 3\left(1+\cos 2 \theta^{\prime}\right) / 2 \\ 0 & (1-\cos 2 \theta) / 2 & 0 \\ 3\left(1+\cos 2 \theta^{\prime}\right) / 2 & 0 & 3\left(1-\cos 2 \theta^{\prime}\right) / 2\end{array}$

(d) Radiation with right circular polarization

\begin{tabular}{lll}
0 & $3(1-\cos 2 \theta) / 2$ & 0 \\
$\left(1+\cos 2 \theta^{\prime}\right) / 2$ & 0 & $\left(1-\cos 2 \theta^{\prime}\right) / 2$ \\
0 & $3(1+\cos 2 \theta) / 2$ & 0 \\
$\left(1-\cos 2 \theta^{\prime}\right) / 2$ & 0 & $\left(1+\cos 2 \theta^{\prime}\right) / 2$ \\
\hline
\end{tabular}


These expansions suffice for listing the intensities where the radiation is linearly polarized parallel to one of the coordinate axes, and results for the field and stress directions are listed in Table III. However, if the polarization is in the $x-y$ plane but not parallel to a coordinate axis, interference occurs between the amplitudes $f_{T}(\theta, \phi)$ and $f_{T}(-\theta,-\phi)$ and another parameter is needed, here designated $w$. It is defined through the relation

$$
D D^{*}-D^{*} D^{\prime}=2 i w N \text {. }
$$

Let the polarization direction be

$$
\widehat{\mathbf{q}}=\hat{\mathbf{x}} \cos \eta+\hat{\mathbf{y}} \sin \eta,
$$

then

$\widehat{\mathbf{q}} \cdot \mathbf{F}(\widehat{\mathbf{x}}, i \widehat{\mathbf{y}} ; \theta, \phi)$

$$
=\cos \eta f_{T}(\theta, \phi)+i \sin \eta f_{T}(-\theta,-\phi),
$$

and

$$
\begin{aligned}
|\widehat{\mathbf{q}} \cdot \mathbf{F}(\widehat{\mathbf{x}}, i \widehat{\mathbf{y}} ; \theta, \phi)|^{2}= & \cos ^{2} \eta\left|f_{T}(\theta, \phi)\right|^{2}+\sin ^{2} \eta\left|f_{T}(-\theta,-\phi)\right|^{2} \\
& +\frac{i}{2} \sin 2 \eta\left[f_{T}^{*}(\theta, \phi) f_{T}(-\theta,-\phi)-f_{T}(\theta, \phi) f_{T}^{*}(-\theta,-\phi)\right] .
\end{aligned}
$$

TABLE III. Prototype display for $\Gamma_{8} \rightarrow \Gamma_{8}\left(\bar{T}_{d}\right)$ transitions under combined stress and magnetic field. (a) For case 1 (B\|[001], $\mathbf{T} \|[100])$ the amplitude parameters $f_{B}, f_{T}, f_{-}$, and $f_{+}$are defined in Eqs. (29), (30), (45), and (46), respectively, and their square

\begin{tabular}{|c|c|c|c|c|c|}
\hline$\mu\left(\bar{T}_{d}\right)$ & $\bar{C}_{2}$ & $\Gamma_{4}$ & $\Gamma_{3}$ & $\Gamma_{4^{\prime}}$ & $\Gamma_{3^{\prime}}$ \\
\hline & & \multicolumn{4}{|c|}{ (a) Radiation polarized parallel to stress } \\
\hline$\frac{3}{2}$ and $-\frac{1}{2}$ & $\Gamma_{4}$ & 0 & $\left|f_{T}\left(\theta^{\prime}, \phi\right)\right|^{2}$ & 0 & $\left|f_{T}\left(\theta^{\prime}+\pi / 2, \phi\right)\right|^{2}$ \\
\hline$\frac{1}{2}$ and $-\frac{3}{2}$ & $\Gamma_{3}$ & $\left|f_{T}\right| \frac{\pi}{2}-\theta, \frac{\pi}{2}-\phi^{\prime}||^{2}$ & 0 & $f_{T} \mid-\theta, \frac{\pi}{2}-\phi^{\prime}$ & 0 \\
\hline$\frac{3}{2}$ and $-\frac{1}{2}$ & $\Gamma_{4^{\prime}}$ & 0 & $\left|f_{T}\left(\theta^{\prime}, \phi+\pi / 2\right)\right|^{2}$ & 0 & $\left|f_{T}\left(\theta^{\prime}+\pi / 2, \phi+\pi / 2\right)\right|^{2}$ \\
\hline$\frac{1}{2}$ and $-\frac{3}{2}$ & $\Gamma_{3^{\prime}}$ & $f_{T}\left[\frac{\pi}{2}-\theta,-\phi^{\prime} \mid\right.$ & 0 & $\left|f_{T}\left(-\theta,-\phi^{\prime}\right)\right|^{2}$ & 0 \\
\hline & & & \multicolumn{3}{|c|}{ (b) Radiation polarized parallel to magnetic field } \\
\hline$\frac{3}{2}$ and $-\frac{1}{2}$ & $\Gamma_{4}$ & $\left|f_{B}(\theta, \phi)\right|^{2}$ & 0 & $\left|f_{B}(\theta+\pi / 2, \phi)\right|^{2}$ & 0 \\
\hline$\frac{1}{2}$ and $-\frac{3}{2}$ & $\Gamma_{3}$ & 0 & $\left|f_{B}\left(\theta^{\prime}-\pi / 2, \phi^{\prime}-\pi / 2\right)\right|^{2}$ & 0 & $\left|f_{B}\left(\theta^{\prime}, \phi^{\prime}-\pi / 2\right)\right|^{2}$ \\
\hline$\frac{3}{2}$ and $-\frac{1}{2}$ & $\Gamma_{4^{\prime}}$ & $\left|f_{B}(\theta, \phi+\pi / 2)\right|^{2}$ & 0 & $\left|f_{B}(\theta+\pi / 2, \phi+\pi / 2)\right|^{2}$ & 0 \\
\hline$\frac{1}{2}$ and $-\frac{3}{2}$ & $\Gamma_{3^{\prime}}$ & 0 & $\left|f_{B}\left(\theta^{\prime}-\pi / 2, \phi^{\prime}\right)\right|^{2}$ & 0 & $\left|f_{B}\left(\theta^{\prime}, \phi^{\prime}\right)\right|^{2}$ \\
\hline & & \multicolumn{4}{|c|}{ (c) Radiation with left circular polarization } \\
\hline$\frac{3}{2}$ and $-\frac{1}{2}$ & $\Gamma_{4}$ & 0 & $\left|f_{-}\left(\theta^{\prime}, \phi\right)\right|^{2}$ & 0 & $\left|f_{-}\left(\theta^{\prime}+\pi / 2, \phi\right)\right|^{2}$ \\
\hline$\frac{1}{2}$ and $-\frac{3}{2}$ & $\Gamma_{3}$ & $\left|f_{+}\left(\theta-\pi / 2, \phi^{\prime}-\pi / 2\right)\right|^{2}$ & 0 & $\left|f_{+}\left(\theta, \phi^{\prime}-\pi / 2\right)\right|^{2}$ & 0 \\
\hline$\frac{3}{2}$ and $-\frac{1}{2}$ & $\Gamma_{4^{\prime}}$ & 0 & $\left|f_{-}\left(\theta^{\prime}, \phi+\pi / 2\right)\right|^{2}$ & 0 & $\left|f_{-}\left(\theta^{\prime}+\pi / 2, \phi+\pi / 2\right)\right|^{2}$ \\
\hline$\frac{1}{2}$ and $-\frac{3}{2}$ & $\Gamma_{3^{\prime}}$ & $\left|f_{+}\left(\theta-\pi / 2, \phi^{\prime}\right)\right|^{2}$ & 0 & $\left|f_{+}\left(\theta, \phi^{\prime}\right)\right|^{2}$ & 0 \\
\hline & & \multicolumn{4}{|c|}{ (d) Radiation with right circular polarization } \\
\hline$\frac{3}{2}$ and $-\frac{1}{2}$ & $\Gamma_{4}$ & 0 & $\left|f_{+}\left(\theta^{\prime}, \phi\right)\right|^{2}$ & 0 & $\left|f_{+}\left(\theta^{\prime}+\pi / 2, \phi\right)\right|^{2}$ \\
\hline$\frac{1}{2}$ and $-\frac{3}{2}$ & $\Gamma_{3}$ & $\left|f_{-}\left(\theta-\pi / 2, \phi^{\prime}-\pi / 2\right)\right|^{2}$ & 0 & $\left|f_{-}\left(\theta, \phi^{\prime}-\pi / 2\right)\right|^{2}$ & 0 \\
\hline$\frac{3}{2}$ and $-\frac{1}{2}$ & $\Gamma_{4^{\prime}}$ & 0 & $\left|f_{+}\left(\theta^{\prime}, \phi+\pi / 2\right)\right|^{2}$ & 0 & $\left|f_{+}\left(\theta^{\prime}+\pi / 2, \phi+\pi / 2\right)\right|^{2}$ \\
\hline$\frac{1}{2}$ and $-\frac{3}{2}$ & $\Gamma_{3^{\prime}}$ & $\left|f_{-}\left(\theta-\pi / 2, \phi^{\prime}\right)\right|^{2}$ & 0 & $\left|f_{-}\left(\theta, \phi^{\prime}\right)\right|^{2}$ & 0 \\
\hline
\end{tabular}
magnitudes expanded in terms of their arguments and the traditional parameters $u$ and $v$ in equations (36), (37), (47), and (48), respectively. (b) For case $2(\mathbf{B} \|[001]$, $\mathbf{T} \|[110])$, the amplitudes $f_{B}, f_{T}, f_{-}$, and $f_{+}$should be replaced by $f_{B}^{\prime}, f_{T}^{\prime}, f_{-}^{\prime}$, and $f_{+}^{\prime}$ defined in Eqs. (55), (56), (61), and (62), with square magnitude expansions given in Eqs. (63), (64), (70), and (71), and redefinition (52) replaces Eq. (20). 
Use of definitions (30) and (38) leads directly to

$$
\begin{aligned}
i\left[f_{T}^{*}(\theta, \phi) f_{T}(-\theta,-\phi)\right. & \left.-f_{T}(\theta, \phi) f_{T}^{*}(-\theta,-\phi)\right] \\
& =2 \sqrt{3} w N(\sin 2 \theta-\sin 2 \phi),
\end{aligned}
$$

so that

$$
\begin{aligned}
\left.\hat{\mathbf{q}} \cdot \mathbf{F}(\hat{\mathbf{x}}, \mathrm{i} \hat{\mathbf{y}} ; \theta, \phi)\right|^{2} \\
=\cos ^{2} \eta\left|f_{T}(\theta, \phi)\right|^{2}+\sin ^{2} \eta\left|f_{T}(-\theta,-\phi)\right|^{2} \\
\quad+\sqrt{3} w N \sin 2 \eta(\sin 2 \theta-\sin 2 \phi)
\end{aligned}
$$

and

$$
\begin{aligned}
|\widehat{\mathbf{q}} \cdot \mathbf{F}(i \widehat{\mathbf{y}},-\widehat{\mathbf{x}} ; \theta, \phi)|^{2} & \\
= & \sin ^{2} \eta\left|f_{T}(\theta, \phi)\right|^{2}+\cos ^{2} \eta\left|f_{T}(-\theta,-\phi)\right|^{2} \\
& +\sqrt{3} w N \sin 2 \eta(\sin 2 \theta-\sin 2 \phi) .
\end{aligned}
$$

Thus, provided $\sin 2 \theta$ and $\sin 2 \phi$ are sufficiently different, $w$ is accessible to measurement. For $\sin 2 \theta$ and $\sin 2 \theta^{\prime}$ to be nonvanishing, $h_{3}$ and thereby the stress must be nonvanishing, with similar considerations for $\phi$ and $\phi^{\prime}$, so for this case a minimum condition for $w$ to be observed is the presence of stress. It is easily seen that a magnetic field must also be present, for if it were not, each of $\sin 2 \theta$ and $\sin 2 \phi$ would be equal to $\sqrt{3} / 2$ independent of stress, and the coefficient of $w$ in Eqs. (43) and (44) would vanish.

Together, Eqs. (43) and (44), perhaps with some guidance from the matrix of Eq. (32), allow the extension of the relevant results of Table III to any plane polarization in the $x-y$ plane. If the polarization has a component parallel to the magnetic field, there is no additional interference between the amplitudes, simply a resolution into components with the intensities given by the foregoing considerations.

For radiation propagating parallel to the magnetic field, the intensities for circular polarization can be expressed in terms of $N, u$, and $v$ without any need for $w$. For convenience in tabulation, we define two more amplitude functions:

$$
\begin{aligned}
& f_{-}(\theta, \phi)=\frac{1}{\sqrt{2}}\left[f_{T}(\theta, \phi)-f_{T}(-\theta,-\phi)\right], \\
& f_{+}(\theta, \phi)=\frac{1}{\sqrt{2}}\left[f_{T}(\theta, \phi)+f_{T}(-\theta,-\phi)\right] .
\end{aligned}
$$

Then

$$
\begin{aligned}
\left|\widehat{\mathbf{q}}_{L} \cdot \mathbf{F}(\widehat{\mathbf{x}}, i \widehat{\mathbf{y}} ; \theta, \phi)\right|^{2} & =\left|f_{-}(\theta, \phi)\right|^{2} \\
& =\frac{3}{16} N u[1-\cos (2 \theta-2 \phi)],
\end{aligned}
$$

and

$$
\begin{aligned}
\left|\widehat{\mathbf{q}}_{R} \cdot \mathbf{F}(\widehat{\mathbf{x}}, i \widehat{\mathbf{y}} ; \theta, \phi)\right|^{2} & =\left|f_{+}(\theta, \phi)\right|^{2} \\
& =\frac{N}{4}\left\{\left[1-\frac{3 u}{4}\right][1+\cos (2 \theta+2 \phi)]-\frac{u}{2} \sin 2 \theta \sin 2 \phi-v(\cos 2 \theta+\cos 2 \phi)\right\} .
\end{aligned}
$$

Also,

$$
\left|\widehat{\mathbf{q}}_{L} \cdot \mathbf{F}(i \widehat{\mathbf{y}},-\widehat{\mathbf{x}} ; \theta, \phi)\right|^{2}=\left|f_{+}(\theta, \phi)\right|^{2}
$$

and

$$
\left|\widehat{\mathbf{q}}_{R} \cdot \mathbf{F}(i \widehat{\mathbf{y}},-\widehat{\mathbf{x}} ; \theta, \phi)\right|^{2}=\left|f_{-}(\theta, \phi)\right|^{2} .
$$

Intensities for circular polarization have been included in Table III.

\section{B. Case 2: stress parallel to [110], magnetic field parallel to [001]}

\section{Energies and wave functions}

As for case 1 , the symmetry group is $\bar{C}_{2}$, and the identification of the symmetry labels for the states under perturbation are as previously derived. The Zeeman Hamiltonians are the same as for the previous case, and the piezoHamiltonians for the $\Gamma_{6}\left(\bar{T}_{d}\right)$ and $\Gamma_{7}\left(\bar{T}_{d}\right)$ states are also unaltered, although there is change for the $\Gamma_{8}$ states. For this case the strain components are $\varepsilon_{z z}=s_{12} T, \varepsilon_{x x}=\varepsilon_{y y}=\frac{1}{2}\left(s_{11}+s_{12}\right) T, \varepsilon_{x y}=\frac{1}{4} T s_{44}$, and $\varepsilon_{y z}=\varepsilon_{z x}=0$. The $\Gamma_{8}$ piezoHamiltonian is

$$
H_{p}^{(8)}=a^{\prime}\left(s_{11}+2 s_{12}\right) T I-\frac{1}{2} b^{\prime}\left(s_{11}-s_{12}\right) T\left(J_{z}^{2}-\frac{5}{4} I\right)+\frac{d^{\prime}}{2 \sqrt{3}} s_{44} T\left\{J_{x} J_{y}\right\} .
$$

The explicit matrix expression for the sum of the stress and Zeeman Hamiltonians is as shown in Eq. (16) subject to the single redefinition

$$
h_{3}=h_{3}^{\prime}=i s_{44} T d^{\prime} / 4 \text {. }
$$

The unitary transformation that diagonalizes the interaction Hamiltonian is again that of Eq. (22). The definitions of $\alpha$, 
TABLE IV. $\Gamma_{8} \rightarrow \Gamma_{6}$ and $\Gamma_{8} \rightarrow \Gamma_{7}$ transitions. Relative intensities for magnetic field parallel to [001] and stress parallel to [110]. The radiation is polarized parallel to the stress. Angles $2 \theta$ and $2 \theta^{\prime}$ are defined in terms of field, stress, and coupling parameters in Eqs. (24) and (25), respectively, but the parameter $h_{3}$ entering those definitions is redefined in Eq. (52). For radiation polarized parallel to the magnetic field, and for left and right circular polarization, see the entries of Table II.

\begin{tabular}{llllll}
\multicolumn{1}{c}{$T_{d}$} & $C_{2}$ & \multicolumn{1}{c}{$\Gamma_{4}$} & \multicolumn{1}{c}{$\Gamma_{3}$} & \multicolumn{1}{c}{$\Gamma_{4^{\prime}}$} & \multicolumn{1}{c}{$\Gamma_{3^{\prime}}$} \\
\hline$\Gamma_{6}\left(\frac{1}{2}\right)$ & $\Gamma_{3}$ & $1+\cos (2 \theta+\pi / 3)$ & 0 & $1-\cos (2 \theta+\pi / 3)$ & 0 \\
$\Gamma_{6}\left(-\frac{1}{2}\right)$ & $\Gamma_{4}$ & 0 & $1-\cos \left(2 \theta^{\prime}-\pi / 3\right)$ & 0 & $1+\cos \left(2 \theta^{\prime}-\pi / 3\right)$ \\
$\Gamma_{7}\left(\frac{1}{2}\right)$ & $\Gamma_{3}$ & $1-\cos (2 \theta-\pi / 3)$ & 0 & $1+\cos (2 \theta-\pi / 3)$ & 0 \\
$\Gamma_{7}\left(-\frac{1}{2}\right)$ & $\Gamma_{4}$ & 0 & $1+\cos \left(2 \theta^{\prime}+\pi / 3\right)$ & 0 & $1-\cos \left(2 \theta^{\prime}+\pi / 3\right)$ \\
\hline \hline
\end{tabular}

$\alpha^{\prime}, \theta$, and $\theta^{\prime}$ given in Eqs. (23) $-(25)$, and the energies of Eqs. (26a) $-(26 \mathrm{~d})$, are appropriate here provided that on each appearance $h_{3}$ and $h_{3}^{\prime}$ are replaced by the redefined values of Eq. (52).

\section{Intensities}

a. $\Gamma_{8} \rightarrow \Gamma_{6}$ transitions. As before, we proceed by constructing $\underline{Q}^{(8 \rightarrow 6)} S$ where these factors are given in Eqs. (7) and (22), respectively, to obtain

$$
\underline{Q}^{(8 \rightarrow 6)} S=D_{0}\left(\begin{array}{cccc}
\sqrt{3}(\widehat{\mathbf{x}}+i \widehat{\mathbf{y}}) \cos \theta & -2 i \widehat{\mathbf{z}} \cos \theta^{\prime} & -i \sqrt{3}(\widehat{\mathbf{x}}+i \widehat{\mathbf{y}}) \sin \theta & -2 \widehat{\mathbf{z}} \sin \theta^{\prime} \\
-i(\hat{\mathbf{x}}-i \widehat{\mathbf{y}}) \sin \theta & & +(\widehat{\mathbf{x}}-i \widehat{\mathbf{y}}) \cos \theta & \\
-2 i \widehat{\mathbf{z}} \sin \theta & i(\widehat{\mathbf{x}}+i \widehat{\mathbf{y}}) \cos \theta^{\prime} & 2 \widehat{\mathbf{z}} \cos \theta & i \sqrt{3}(\widehat{\mathbf{x}}-i \widehat{\mathbf{y}}) \cos \theta^{\prime} \\
& +\sqrt{3}(\widehat{\mathbf{x}}-i \widehat{\mathbf{y}}) \sin \theta^{\prime} & & +(\widehat{\mathbf{x}}+i \widehat{\mathbf{y}}) \sin \theta^{\prime}
\end{array}\right) .
$$

For linear polarization parallel to the stress, the intensities arising from these amplitudes are listed in Table IV. For polarization parallel to the magnetic field and for circular polarization, the entries of Table II are applicable subject to the change in the definition of the parameter $h_{3}$.

b. $\Gamma_{8} \rightarrow \Gamma_{7}$ transitions. The amplitude matrix is formed by using Eqs. (8) and (22), yielding

$$
\underline{Q}^{(8 \rightarrow 7)} S=D_{0}^{\prime}\left(\begin{array}{cccc}
-(\hat{\mathbf{x}}-i \widehat{\mathbf{y}}) \cos \theta & 2 \widehat{\mathbf{z}} \sin \theta^{\prime} & i(\widehat{\mathbf{x}}-i \widehat{\mathbf{y}}) \sin \theta & 2 i \widehat{\mathbf{z}} \cos \theta^{\prime} \\
-i \sqrt{3}(\widehat{\mathbf{x}}+i \widehat{\mathbf{y}}) \sin \theta & & +\sqrt{3}(\widehat{\mathbf{x}}+i \hat{\mathbf{y}}) \cos \theta & \\
-2 \widehat{\mathbf{z}} \cos \theta & i \sqrt{3}(\widehat{\mathbf{x}}-i \widehat{\mathbf{y}}) \cos \theta^{\prime} & 2 i \widehat{\mathbf{z}} \sin \theta & \sqrt{3}(\widehat{\mathbf{x}}-i \widehat{\mathbf{y}}) \sin \theta^{\prime} \\
& -(\widehat{\mathbf{x}}+i \widehat{\mathbf{y}}) \sin \theta^{\prime} & & -i(\widehat{\mathbf{x}}+i \widehat{\mathbf{y}}) \cos \theta^{\prime}
\end{array}\right) .
$$

The intensities arising from these amplitudes are incorporated in Table IV.

c. $\Gamma_{8} \rightarrow \Gamma_{8}$ transitions. Once more the procedure for forming the transition amplitudes must recognize that, in contrast to the $\Gamma_{8} \rightarrow \Gamma_{6}$ and $\Gamma_{8} \rightarrow \Gamma_{7}$ transitions, both the bra and the ket states have been mixed in the presence of the stress and magnetic field, so $S_{2}^{\dagger}\left(\phi, \phi^{\prime}\right) \underline{Q}^{8 \rightarrow}{ }^{8} S_{1}\left(\theta, \theta^{\prime}\right)$ must be constructed; in both sets of parameters the redefinition (52) applies. It is convenient to introduce some amplitude parameters analogous to those of Eqs. (29) $-(31)$. These are

$$
\begin{aligned}
& f_{B}^{\prime}(\theta, \phi)=\left(D+3 D^{\prime}\right) \cos \theta \sin \phi+\left(D-D^{\prime}\right) \sin \theta \cos \phi, \\
& f_{T}^{\prime}(\theta, \phi)=D \sin \theta \sin \phi-\sqrt{3} D^{\prime}(\sin \theta \cos \phi+\cos \theta \sin \phi)-\left(D+2 D^{\prime}\right) \cos \theta \cos \phi,
\end{aligned}
$$

and

$$
F^{\prime}(\hat{\mathbf{a}}, \hat{\mathbf{b}} ; \theta, \phi)=\widehat{\mathbf{a}} f_{T}^{\prime}(\theta, \phi)+\widehat{\mathbf{b}} f_{T}^{\prime}(-\theta,-\phi) .
$$

Also, it is useful to specify symbols for two unit vectors orthogonal to the magnetic-field direction $\widehat{\mathbf{z}}$, one of them parallel to the stress. These are

$$
\hat{\mathbf{x}}=\frac{1}{\sqrt{2}}(\hat{\mathbf{x}}+\hat{\mathbf{y}})
$$

and

$$
\widehat{\mathbf{Y}}=\frac{1}{\sqrt{2}}(\hat{\mathbf{y}}-\hat{\mathbf{x}})
$$

The amplitudes are as follows: 
Apart from the overall phase factors, there is strong formal similarity between these amplitudes and those of Eq. (32). It follows that the intensities of Table III are applicable here provided that the amplitudes $f_{B}$ and $f_{T}$ used therein are replaced, respectively, by $f_{B}^{\prime}$ and $f_{T}^{\prime}$ defined in Eqs. (55) and (56), and definitions analogous to those of Eqs. (45) and (46) are introduced to describe circular polarization for the present case, replacing those used in Table III.

$$
\begin{aligned}
& f_{-}^{\prime}(\theta, \phi)=\frac{1}{\sqrt{2}}\left[f_{T}^{\prime}(\theta, \phi)-f_{T}^{\prime}(-\theta,-\phi)\right], \\
& f_{+}^{\prime}(\theta, \phi)=\frac{1}{\sqrt{2}}\left[f_{T}^{\prime}(\theta, \phi)+f_{T}^{\prime}(-\theta,-\phi)\right] .
\end{aligned}
$$

The intensities for linear polarization are expressed in terms of $\left|f_{B}^{\prime}\right|^{2}$ and $\left|f_{T}^{\prime}\right|^{2}$, the explicit expansions of which are as follows:

$$
\begin{aligned}
\left|f_{B}^{\prime}(\theta, \phi)\right|^{2}= & \frac{N}{8}\{1-\cos (2 \theta+2 \phi)-2 u \sin 2 \theta \sin 2 \phi \\
& +2 v[\cos 2 \phi-\cos 2 \theta]\} \\
\left|f_{T}^{\prime}(\theta, \phi)\right|^{2}= & \frac{N}{8}[1+\cos (2 \theta+2 \phi)] \\
& -\frac{N v}{8}[\cos 2 \theta+\cos 2 \phi+\sqrt{3} \sin (2 \theta+2 \phi)] \\
+ & \frac{N u}{16}[4 \sin 2 \theta \sin 2 \phi-3 \cos 2 \theta \cos 2 \phi \\
& \quad+\sqrt{3}(\sin 2 \theta+\sin 2 \phi)] .
\end{aligned}
$$

Again the parameter $w$ appears for linear polarization in the $X Y$ plane neither parallel nor orthogonal to the stress. Thus if the polarization is

$$
\widehat{\mathbf{q}}=\hat{\mathbf{X}} \cos \eta+\widehat{\mathbf{Y}} \sin \eta,
$$

then

$$
\begin{aligned}
\left|\widehat{\mathbf{q}} \cdot \mathbf{F}^{\prime}(\hat{\mathbf{X}}, i \hat{\mathbf{Y}} ; \theta, \phi)\right|^{2} & \\
= & \cos ^{2} \eta\left|f_{T}^{\prime}(\theta, \phi)\right|^{2}+\sin ^{2} \eta\left|f_{T}^{\prime}(-\theta,-\phi)\right|^{2} \\
& -\sqrt{3} w N \sin (2 \theta+2 \phi) \sin 2 \eta, \\
\left|\widehat{\mathbf{q}} \cdot \mathbf{F}^{\prime}(i \hat{\mathbf{Y}},-\hat{\mathbf{X}} ; \theta, \phi)\right|^{2} & \\
= & \sin ^{2} \eta\left|f_{T}^{\prime}(\theta, \phi)\right|^{2}+\cos ^{2} \eta\left|f_{T}^{\prime}(-\theta,-\phi)\right|^{2} \\
& -\sqrt{3} w N \sin (2 \theta+2 \phi) \sin 2 \eta .
\end{aligned}
$$

If stress is absent, both $\theta$ and $\phi$ are zero, so the coefficient of $w$ in Eqs. (66) and (67) is likewise zero. If the magnetic field is zero, then the states occur in degenerate pairs, and in the sum of intensities for equal-energy transitions there is a cancellation of the terms containing $w$. Thus again, both stress and field are needed for $w$ to be observed.

For left and right circular polarization, the polarization vectors $\widehat{\mathbf{q}}_{L}$ and $\hat{\mathbf{q}}_{R}$ can be written

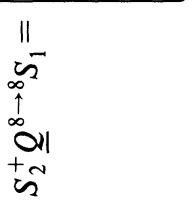

and

$$
\widehat{\mathbf{q}}_{L}=(\hat{\mathbf{x}}+i \hat{\mathbf{y}}) / \sqrt{2}=e^{i \pi / 4}(\hat{\mathbf{X}}+i \hat{\mathbf{Y}})
$$$$
\widehat{\mathbf{q}}_{R}=(\widehat{\mathbf{x}}-i \widehat{\mathbf{y}}) / \sqrt{2}=e^{-i \pi / 4}(\hat{X}-i \hat{\mathbf{Y}}) .
$$

The intensities for circular polarization are then 


$$
\begin{aligned}
\left|\widehat{\mathbf{q}}_{L} \cdot \mathbf{F}^{\prime}(\hat{\mathbf{X}}, i \hat{\mathbf{Y}} ; \theta, \phi)\right|^{2} & =\left|\widehat{\mathbf{q}}_{R} \cdot \mathbf{F}^{\prime}(i \hat{\mathbf{Y}},-\hat{\mathbf{X}} ; \theta, \phi)\right|^{2} \\
& =\left|f_{-}^{\prime}(\theta, \phi)\right|^{2} \\
& =\frac{3}{16} N u[1-\cos (2 \theta+2 \phi)], \\
\left|\hat{\mathbf{q}}_{R} \cdot \mathbf{F}^{\prime}(\widehat{\mathbf{X}}, i \hat{\mathbf{Y}} ; \theta, \phi)\right|^{2} & =\left|\hat{\mathbf{q}}_{L} \cdot \mathbf{F}^{\prime}(i \hat{\mathbf{Y}},-\hat{\mathbf{X}} ; \theta, \phi)\right|^{2} \\
& =\left|f_{+}^{\prime}(\theta, \phi)\right|^{2} \\
& =\frac{N}{4}\left\{\left[1-\frac{3 u}{4}\right][1+\cos (2 \theta+2 \phi)]-\frac{u}{2} \sin 2 \theta \sin 2 \phi-v(\cos 2 \theta+\cos 2 \phi)\right\} .
\end{aligned}
$$

\section{Case 3: stress parallel to [001], magnetic field parallel to [ $\overline{1} 10]$}

\section{Energies and wave functions}

The strain components for the present case are $\varepsilon_{z z}=s_{11} T, \varepsilon_{x x}=\varepsilon_{y y}=s_{12} T$, and $\varepsilon_{x y}=\varepsilon_{y z}=\varepsilon_{z x}=0$, and the magnetic field is $\mathbf{B}=\boldsymbol{B}(\hat{\mathbf{y}}-\hat{\mathbf{x}}) / \sqrt{2}$. The only crystal symmetry elements shared by both the stress and the magnetic field are $E, \bar{E}, \sigma$, and $\bar{\sigma}$, so the symmetry group is $C_{s}$. The piezo-Hamiltonians are analogs of those used for case 1, and the Zeeman Hamiltonians are obtained directly from Eqs. (2) and (3); these are

$H_{p}^{(6,7)}=a^{(6,7)}\left(s_{11}+2 s_{12}\right) T I$,

$H_{p}^{(8)}=a^{\prime}\left(s_{11}+2 s_{12}\right) T I+b^{\prime}\left(s_{11}-s_{12}\right) T\left(J_{z}^{2}-\frac{5}{4} I\right)$,

$H_{Z}^{(6,7)}=\mu_{B} g^{(6,7)} B\left(j_{y}-j_{x}\right) / \sqrt{2}+q^{(6,7)} B^{2} I$,

and

$$
\begin{aligned}
H_{Z}^{(8)}= & \mu_{B} \frac{B}{\sqrt{2}}\left[g_{1}^{\prime}\left(J_{y}-J_{x}\right)+g_{2}^{\prime}\left(J_{y}^{3}-J_{x}^{3}\right)\right] \\
& +\frac{B^{2}}{2}\left[2 q_{1}+q_{2}\left(J_{y}-J_{x}\right)^{2}+q_{3}\left(J_{x}^{2}+J_{y}^{2}\right)\right] .
\end{aligned}
$$

Suitable rotated functions for the initial state of diagonalization of the combined piezo-Hamiltonians and Zeeman Hamiltonians are achieved in two convenient steps, first a rotation by $\pi / 2$ about the $y$ axis, then a second rotation of $3 \pi / 4$ about the $z$ axis. This is all that is required for the $\Gamma_{6}\left(\bar{T}_{d}\right)$ and $\Gamma_{7}\left(\bar{T}_{d}\right)$ states. The resulting functions, their symmetries and energy eigenvalues are as follows.

For $\Gamma_{3}\left(C_{s}\right)$,

$\Psi^{(3)}=\frac{1}{\sqrt{2}}\left[e^{i 3 \pi / 8} \psi_{-1 / 2}^{(6)}+e^{-i 3 \pi / 8} \psi_{1 / 2}^{(6)}\right]$,

$E\left(\Psi^{(3)}\right)=a^{(6)}\left(s_{11}+2 s_{12}\right) T+\mu_{B} g^{(6)} \frac{B}{2}+q^{(6)} B^{2}$

$\Psi^{\left(3^{\prime}\right)}=\frac{1}{\sqrt{2}}\left[e^{i 3 \pi / 8} \psi_{-1 / 2}^{(7)}-e^{-i 3 \pi / 8} \psi_{1 / 2}^{(7)}\right]$,

$E\left(\Psi^{\left(3^{\prime}\right)}\right)=a^{(7)}\left(s_{11}+2 s_{12}\right) T-\mu_{B} g^{(7)} \frac{B}{2}+q^{(7)} B^{2}$.

For $\Gamma_{4}\left(C_{s}\right)$,

$\Psi^{(4)}=\frac{1}{\sqrt{2}}\left[e^{i 3 \pi / 8} \psi_{-1 / 2}^{(6)}-e^{-i 3 \pi / 8} \psi_{1 / 2}^{(6)}\right]$,

$E\left(\Psi^{(4)}\right)=a^{(6)}\left(s_{11}+2 s_{12}\right) T-\mu_{B} g^{(6)} \frac{B}{2}+q^{(6)} B^{2}$,

$\Psi^{\left(4^{\prime}\right)}=\frac{1}{\sqrt{2}}\left[e^{i 3 \pi / 8} \psi_{-1 / 2}^{(7)}+e^{-i 3 \pi / 8} \psi_{1 / 2}^{(7)}\right]$,

$E\left(\Psi^{\left(4^{\prime}\right)}\right)=a^{(7)}\left(s_{11}+2 s_{12}\right) T+\mu_{B} g^{(7)} \frac{B}{2}+q^{(7)} B^{2}$.

For $\Gamma_{8}$ states, suitable rotated functions for the first stage of diagonalization are

$$
\Psi_{\mu}=\sum_{\mu^{\prime}} \psi_{\mu^{\prime}}^{(8)}\left[e^{-i(3 \pi / 4) J_{z}} e^{-i(\pi / 2) J_{y}}\right]_{\mu^{\prime} \mu},
$$

where

$$
e^{-i(3 \pi / 4) J_{z}} e^{-i(\pi / 2) J_{y}}=\frac{1}{2 \sqrt{2}}\left(\begin{array}{cccc}
a^{-3} & -i \sqrt{3} a^{-3} & -\sqrt{3} a^{-3} & i a^{-3} \\
-i \sqrt{3} a^{-1} & -a^{-1} & -i a^{-1} & -\sqrt{3} a^{-1} \\
-\sqrt{3} a & -i a & -a & -i \sqrt{3} a \\
i a^{3} & -\sqrt{3} a^{3} & -i \sqrt{3} a^{3} & a^{3}
\end{array}\right)
$$

with $a=e^{i 3 \pi / 8}$. The symmetries of these functions may be determined by consideration of a reflection in a plane perpendicular to the magnetic field. For $\mu=\frac{3}{2}, \frac{1}{2},-\frac{1}{2}$, and $-\frac{3}{2}$ the symmetries of these functions in sequence are $\Gamma_{3}, \Gamma_{4}, \Gamma_{3}$, and $\Gamma_{4}$, and so they are respectively designated $\psi_{3}, \psi_{4}, \psi_{3^{\prime}}$, and $\psi_{4^{\prime}}$. The transformed Hamiltonian

$$
e^{i(\pi / 2) J_{y}} e^{i(3 \pi / 4) J_{z}}\left(H_{Z}^{(8)}+H_{p}^{(8)}\right) e^{-i(3 \pi / 4) J_{z}} e^{-i(\pi / 2) J_{y}}
$$

and has the form given in Eq. (16), where the parameters used therein are here redefined as follows: 
$h_{0}=a^{\prime}\left(s_{11}+2 s_{12}\right) T+B^{2}\left[q_{1}+\frac{5}{4}\left(q_{2}+q_{3}\right)\right]$,

$h_{1}=\mu_{B} B\left(\frac{3}{2} g_{1}^{\prime}+3 g_{2}^{\prime}\right)$,

$h_{2}=\frac{1}{2} b^{\prime}\left(s_{11}-s_{12}\right) T-\left(q_{2}+\frac{1}{4} q_{3}\right) B^{2}$,

$h_{3}=\frac{\sqrt{3}}{2} b^{\prime}\left(s_{12}-s_{11}\right) T+\frac{3 \sqrt{3}}{8} \mu_{B} g_{2}^{\prime} B+\frac{\sqrt{3}}{4} q_{3} B^{2}$,

$h_{3}^{\prime}=\frac{\sqrt{3}}{2} b^{\prime}\left(s_{12}-s_{11}\right) T-\frac{3 \sqrt{3}}{8} \mu_{B} g_{2}^{\prime} B+\frac{\sqrt{3}}{4} q_{3} B^{2}$,

$h_{4}=\mu_{B} B\left(\frac{1}{2} g_{1}^{\prime}+\frac{5}{4} g_{2}^{\prime}\right)$.

It follows that the energy eigenvalues are similar to those of Eqs. (26a)-(26d), but they must be given the correct symmetry labels for the situation at hand:

$$
\begin{aligned}
& E_{3}=h_{0}+\frac{1}{2}\left[h_{1}-h_{4}+\sqrt{\left(h_{1}+h_{4}-2 h_{2}\right)^{2}+4 h_{3}^{2}}\right], \\
& E_{4}=h_{0}+\frac{1}{2}\left[h_{4}-h_{1}+\sqrt{\left(h_{1}+h_{4}+2 h_{2}\right)^{2}+\left(2 h_{3}^{\prime}\right)^{2}}\right], \\
& E_{3^{\prime}}=h_{0}+\frac{1}{2}\left[h_{1}-h_{4}-\sqrt{\left(h_{1}+h_{4}-2 h_{2}\right)^{2}+4 h_{3}^{2}}\right],
\end{aligned}
$$

$$
E_{4^{\prime}}=h_{0}+\frac{1}{2}\left[h_{4}-h_{1}-\sqrt{\left(h_{1}+h_{4}+2 h_{2}\right)^{2}+\left(2 h_{3}^{\prime}\right)^{2}}\right] .
$$

The unitary transformation completing this diagonalization is of course that of Eqs. (22) - (25), with the parameters entering the relations being redefined by Eqs. (83)-(88).

\section{Intensities}

Again it is convenient to describe the polarization vectors in terms of an orthogonal set of unit vectors, one of which is parallel to the magnetic field, and another parallel to the stress, thus

$$
\begin{aligned}
& \hat{\mathbf{X}}=\hat{\mathbf{z}}, \\
& \hat{\mathbf{Y}}=(\widehat{\mathbf{x}}+\hat{\mathbf{y}}) / \sqrt{2}, \\
& \hat{\mathbf{Z}}=(\hat{\mathbf{y}}-\hat{\mathbf{x}}) / \sqrt{2} .
\end{aligned}
$$

Standard procedures applied to matrices (7), (8), and (9) yield the transition amplitudes.

a. $\Gamma_{8} \rightarrow \Gamma_{6}$ transitions. The $2 \times 4$ transition amplitude matrix arising from Eq. (7) is the following:

$$
-D_{0}\left(\begin{array}{cccc}
\sqrt{3}(\hat{\mathbf{X}}+i \hat{\mathbf{Y}}) \cos \theta & 2 i \hat{\mathbf{Z}} \cos \theta^{\prime} & -\sqrt{3}(\hat{\mathbf{X}}+i \hat{\mathbf{Y}}) \sin \theta & -2 i \hat{\mathbf{Z}} \sin \theta^{\prime} \\
+(\hat{\mathbf{X}}-i \hat{\mathbf{Y}}) \sin \theta & & +(\hat{\mathbf{X}}-i \hat{\mathbf{Y}}) \cos \theta & \\
-2 \hat{\mathbf{Z}} \sin \theta & i(\hat{\mathbf{X}}+i \hat{\mathbf{Y}}) \cos \theta^{\prime} & -2 \hat{\mathbf{Z}} \cos \theta & -i(\hat{\mathbf{X}}+i \hat{\mathbf{Y}}) \sin \theta^{\prime} \\
& +i \sqrt{3}(\hat{\mathbf{X}}-i \hat{\mathbf{Y}}) \sin \theta^{\prime} & & +i \sqrt{3}(\hat{\mathbf{X}}-i \hat{\mathbf{Y}}) \cos \theta^{\prime}
\end{array}\right)
$$

The angles $\theta$ and $\theta^{\prime}$ defined in Eqs. (24) and (25) are computed from the set of parameters (83)-(88). Intensities calculated from these amplitudes are listed in Table $\mathrm{V}$.

b. $\quad \Gamma_{8} \rightarrow \Gamma_{7}$ transitions. The $2 \times 4$ transition amplitude matrix constructed from Eq. (8) by use of the appropriate unitary transformations is the following:

$$
2 D_{0}^{\prime}\left(\begin{array}{cccc}
i \hat{\mathbf{Z}} \cos (\theta-\pi / 3) & \hat{\mathbf{X}} \sin \left(\theta^{\prime}-\pi / 3\right) & -i \hat{\mathbf{Z}} \sin (\theta-\pi / 3) & \hat{\mathbf{X}} \cos \left(\theta^{\prime}-\pi / 3\right) \\
& +i \hat{\mathbf{Y}} \sin \theta^{\prime} & & +i \hat{\mathbf{Y}} \cos \theta^{\prime} \\
-i \hat{\mathbf{X}} \cos (\theta+\pi / 3) & \hat{\mathbf{Z}} \sin \left(\theta^{\prime}+\pi / 3\right) & i \hat{\mathbf{X}} \sin (\theta+\pi / 3) & \hat{\mathbf{Z}} \cos \left(\theta^{\prime}+\pi / 3\right) \\
-\hat{\mathbf{Y}} \cos \theta & & +\hat{\mathbf{Y}} \sin \theta &
\end{array}\right)
$$

Intensities arising from these amplitudes are listed in Table V.

c. $\quad \Gamma_{8} \rightarrow \Gamma_{8}$ transitions. The necessary matrix elements are obtained by the construction of $S_{2}^{\dagger}\left(\phi, \phi^{\prime}\right) e^{i(\pi / 2) J_{y}} e^{i(3 \pi / 4) J_{z}} Q^{(8 \rightarrow 8)} e^{-i(3 \pi / 4) J_{z}} e^{-i(\pi / 2) J_{y}} S_{1}\left(\theta, \theta^{\prime}\right)$ in the standard manner. The listing of the results is again assisted by the introduction of some convenient functions, the definitions of which are local to the case being considered.

$$
\begin{aligned}
& f_{B}=-i\left[\left(D+D^{\prime}\right) \cos (\theta+\phi)+\sqrt{3} D^{\prime} \sin (\theta+\phi)-D^{\prime} \cos (\theta-\phi+\pi / 3)\right], \\
& f_{T}=-\left(D+D^{\prime}\right) \sin (\theta+\phi-\pi / 3)-2 D^{\prime} \sin (\theta-\phi), \\
& f_{T}^{\prime}=i\left[D^{\prime} \sin (\theta+\phi-\pi / 3)+\sqrt{3} D^{\prime} \cos (\theta-\phi)-\left(D+D^{\prime}\right) \sin (\theta-\phi)\right], \\
& \mathbf{F}(\widehat{\mathbf{a}}, \hat{\mathbf{b}} ; \theta, \phi)=\hat{\mathbf{a}} f_{T}(\theta, \phi)+\widehat{\mathbf{b}} f_{T}^{\prime}(\theta, \phi) .
\end{aligned}
$$

The transition amplitude matrix expressed in terms of these functions is the following: 


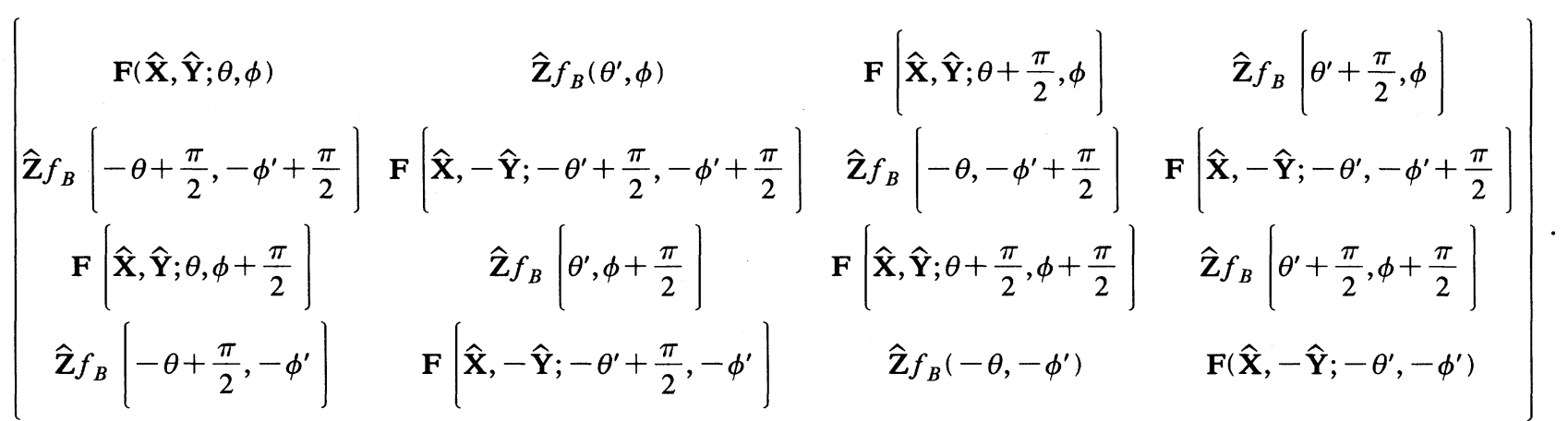

The intensities for linear polarization require the quantities $\left|f_{B}\right|^{2},\left|f_{T}\right|^{2}$, and $\left|f_{T}^{\prime}\right|^{2}$, which are now listed in terms of their arguments and the standard intensity parameters $N, u$, and $v$.

$$
\begin{aligned}
\left|f_{B}(\theta, \phi)\right|^{2}= & \frac{N}{8}\{1+\cos (2 \theta+2 \phi)+v[\cos (2 \theta+\pi / 3)+\cos (2 \phi-\pi / 3)-\sqrt{3} \sin (2 \theta+2 \phi)]\} \\
& +\frac{N u}{64}[6(\cos 2 \phi-\cos 2 \theta)+13 \sin 2 \theta \sin 2 \phi-15 \cos 2 \theta \cos 2 \phi]-\frac{\sqrt{3} N u}{64}[2 \sin 2 \theta+2 \sin 2 \phi+\sin (2 \theta-2 \phi)],
\end{aligned}
$$

TABLE V. $\Gamma_{8} \rightarrow \Gamma_{6}$ and $\Gamma_{8} \rightarrow \Gamma_{7}$ transitions. Relative intensities for $\mathbf{B} \|[\overline{1} 10]$ with $\mathbf{T} \|[001]$. Angles $2 \theta$ and $2 \theta^{\prime}$ are defined in terms of field, stress, and coupling parameters in Eqs. (24) and (25), respectively, but the parameters $h_{1}-h_{4}$ entering those definitions are those defined in Eqs. (84)-(88). The functions indicated in the column headed $\bar{T}_{d}$ are rotated so that the axis of quantization is parallel to the magnetic field, becoming the functions listed in the column headed $\bar{C}_{s}$.

\begin{tabular}{clllll}
\hline $\bar{T}_{d}$ & $\bar{C}_{s}$ & $\Gamma_{3}$ & $\Gamma_{4}$ & $\Gamma_{3^{\prime}}$ & $\Gamma_{4^{\prime}}$ \\
\hline
\end{tabular}

$\begin{array}{lll}\Gamma_{6}\left(\frac{1}{2}\right) & \Gamma_{3} & 1+\cos (2 \theta-\pi / 3) \\ \Gamma_{6}\left(-\frac{1}{2}\right) & \Gamma_{4} & 0 \\ \Gamma_{7}\left(\frac{1}{2}\right) & \Gamma_{4} & 0 \\ \Gamma_{7}\left(-\frac{1}{2}\right) & \Gamma_{3} & 1-\cos (2 \theta-\pi / 3)\end{array}$

$\begin{array}{lll}\Gamma_{6}\left(\frac{1}{2}\right) & \Gamma_{3} & 0 \\ \Gamma_{6}\left(-\frac{1}{2}\right) & \Gamma_{4} & 1-\cos 2 \theta \\ \Gamma_{7}\left(\frac{1}{2}\right) & \Gamma_{4} & 1-\cos (2 \theta+\pi / 3) \\ \Gamma_{7}\left(-\frac{1}{2}\right) & \Gamma_{3} & 0\end{array}$

$\begin{array}{lll}\Gamma_{6}\left(\frac{1}{2}\right) & \Gamma_{3} & (1-\cos 2 \theta) / 2 \\ \Gamma_{6}\left(-\frac{1}{2}\right) & \Gamma_{4} & 0 \\ \Gamma_{7}\left(\frac{1}{2}\right) & \Gamma_{4} & 0 \\ \Gamma_{7}\left(-\frac{1}{2}\right) & \Gamma_{3} & 3[1+\cos (2 \theta+\pi / 3)] / 2\end{array}$

(a) Radiation polarized parallel to stress

$\begin{array}{lll}0 & 1-\cos (2 \theta-\pi / 3) & 0 \\ 1-\cos \left(2 \theta^{\prime}+\pi / 3\right) & 0 & 1+\cos \left(2 \theta^{\prime}+\pi / 3\right) \\ 1+\cos \left(2 \theta^{\prime}+\pi / 3\right) & 0 & 1-\cos \left(2 \theta^{\prime}+\pi / 3\right) \\ 0 & 1+\cos (2 \theta-\pi / 3) & 0\end{array}$

(b) Radiation polarized parallel to magnetic field

$\begin{array}{lll}1+\cos 2 \theta^{\prime} & 0 & 1-\cos 2 \theta^{\prime} \\ 0 & 1+\cos 2 \theta & 0 \\ 0 & 1+\cos (2 \theta+\pi / 3) & 0 \\ 1+\cos \left(2 \theta^{\prime}-\pi / 3\right) & 0 & 1-\cos \left(2 \theta^{\prime}-\pi / 3\right)\end{array}$

(c) Radiation with left circular polarization

$\begin{array}{lll}0 & (1+\cos 2 \theta) / 2 & 0 \\ 3\left(1-\cos 2 \theta^{\prime}\right) / 2 & 0 & 3\left(1+\cos 2 \theta^{\prime}\right) / 2 \\ {\left[1+\cos \left(2 \theta^{\prime}-\pi / 3\right)\right] / 2} & 0 & {\left[1-\cos \left(2 \theta^{\prime}-\pi / 3\right)\right] / 2} \\ 0 & 3[1-\cos (2 \theta+\pi / 3)] / 2 & 0\end{array}$

(d) Radiation with right circular polarization

\begin{tabular}{llllll}
$\Gamma_{6}\left(\frac{1}{2}\right)$ & $\Gamma_{3}$ & $3(1+\cos 2 \theta) / 2$ & 0 & $3(1-\cos 2 \theta) / 2$ & 0 \\
$\Gamma_{6}\left(-\frac{1}{2}\right)$ & $\Gamma_{4}$ & 0 & $\left(1+\cos 2 \theta^{\prime}\right) / 2$ & 0 & $\left(1-\cos 2 \theta^{\prime}\right) / 2$ \\
$\Gamma_{7}\left(\frac{1}{2}\right)$ & $\Gamma_{4}$ & 0 & $3\left[1-\cos \left(2 \theta^{\prime}-\pi / 3\right)\right] / 2$ & 0 & $3\left[1+\cos \left(2 \theta^{\prime}-\pi / 3\right)\right] / 2$ \\
$\Gamma_{7}\left(-\frac{1}{2}\right)$ & $\Gamma_{3}$ & {$[1-\cos (2 \theta+\pi / 3)] / 2$} & 0 & {$[1+\cos (2 \theta+\pi / 3)] / 2$} & 0 \\
\hline \hline
\end{tabular}




$$
\begin{aligned}
& \left|f_{T}(\theta, \phi)\right|^{2}=\frac{N}{8}\{1+\cos (2 \theta+2 \phi+\pi / 3)+u[\cos (2 \theta+2 \phi-\pi / 3)-\cos (2 \theta-2 \phi)] \\
& +4 v[\cos (2 \theta-\pi / 3)-\cos (2 \phi-\pi / 3)]\}, \\
& \left|f_{T}^{\prime}(\theta, \phi)\right|^{2}=\frac{N}{16}\left\{2+\left[v-\frac{3 u}{2}\right] \cos 2 \phi-\left[v+\frac{3 u}{2}\right] \cos 2 \theta-\left(2-\frac{15 u}{4}\right) \cos 2 \theta \cos 2 \phi\right. \\
& +\sqrt{3}\left[\left(v+\frac{u}{2}\right] \sin 2 \phi-\left[v-\frac{u}{2}\right] \sin 2 \theta+\left[2 v-\frac{u}{4}\right] \sin 2 \theta \cos 2 \phi\right. \\
& \left.\left.-\left[2 v+\frac{u}{4}\right] \cos 2 \theta \sin 2 \phi\right]-\left(2-\frac{13 u}{4}\right) \sin 2 \theta \sin 2 \phi\right\} \text {. }
\end{aligned}
$$

For linear polarization in the $\hat{\mathbf{X}} \hat{\mathbf{Y}}$ plane, the parameter $w$ defined in Eq. (37) again makes an appearance. Let the polarization be

$$
\hat{\mathbf{q}}=\hat{\mathbf{X}} \cos \eta+\hat{\mathbf{Y}} \sin \eta
$$

TABLE VI. $\Gamma_{8} \rightarrow \Gamma_{8}\left(\bar{T}_{d}\right)$ transition intensities for $\mathbf{B}\|[\overline{1} 10], \mathbf{T}\|[001]$. The amplitude functions $f_{B}, f_{T}, f_{-}$, and $f_{+}$are defined in Eqs. (95), (96), (107), and (108), respectively, and their square magnitudes expanded in terms of their arguments and the traditional parameters $u$ and $v$ and normalization $N$ in Eqs. (100), (101), and (109)-(111), respectively. The calculation of the angles $\theta, \theta^{\prime}, \phi$, and $\phi^{\prime}$ via Eqs. (24) and (25) require the use of the parameters defined in (84)-(88).

\begin{tabular}{cccccc}
\hline$\mu\left(\bar{T}_{d}\right)$ & $\bar{C}_{s}$ & $\Gamma_{3}$ & $\Gamma_{4}$ & $\Gamma_{3^{\prime}}$ & $\Gamma_{4^{\prime}}$
\end{tabular}

$\begin{array}{lll}\frac{3}{2} \text { and }-\frac{1}{2} & \Gamma_{3} & \left|f_{T}(\theta, \phi)\right|^{2} \\ \frac{1}{2} \text { and }-\frac{3}{2} & \Gamma_{4} & 0 \\ \frac{3}{2} \text { and }-\frac{1}{2} & \Gamma_{3^{\prime}} & \left|f_{T}(\theta, \phi+\pi / 2)\right|^{2} \\ \frac{1}{2} \text { and }-\frac{3}{2} & \Gamma_{4^{\prime}} & 0\end{array}$

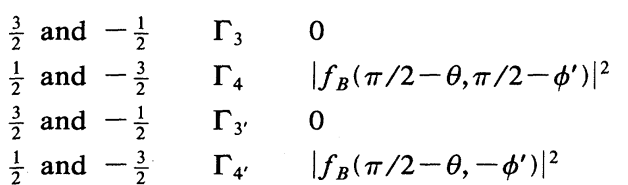

$\frac{3}{2}$ and $-\frac{1}{2} \quad \Gamma_{3} \quad\left|f_{-}(\theta, \phi)\right|^{2}$

$\frac{1}{2}$ and $-\frac{3}{2} \quad \Gamma_{4} \quad 0$

$\frac{3}{2}$ and $-\frac{1}{2} \quad \Gamma_{3^{\prime}} \quad\left|f_{-}(\theta, \phi+\pi / 2)\right|^{2}$

$\frac{1}{2}$ and $-\frac{3}{2} \quad \Gamma_{4^{\prime}} \quad 0$

$\begin{array}{lll}\frac{3}{2} \text { and }-\frac{1}{2} & \Gamma_{3} & \left|f_{+}(\theta, \phi)\right|^{2} \\ \frac{1}{2} \text { and }-\frac{3}{2} & \Gamma_{4} & 0 \\ \frac{3}{2} \text { and }-\frac{1}{2} & \Gamma_{3^{\prime}} & \left|f_{+}(\theta, \phi+\pi / 2)\right|^{2} \\ \frac{1}{2} \text { and }-\frac{3}{2} & \Gamma_{4^{\prime}} & 0\end{array}$

(a) Radiation polarized parallel to stress

$$
\begin{array}{lll}
\left|f_{T}\left(\frac{\pi}{2}-\theta^{\prime}, \frac{\pi}{2}-\phi^{\prime}\right)\right|^{2} & 0 & \left|f_{T}(\theta+\pi / 2, \phi)\right|^{2} \\
0 & \left|f_{T}(\theta+\pi / 2, \phi+\pi / 2)\right|^{2} & 0 \\
\left|f_{T}\left(\frac{\pi}{2}-\theta^{\prime},-\phi^{\prime}\right)\right|^{2} & 0 & \left|f_{T}\left(-\theta^{\prime},-\frac{\pi}{2}-\phi^{\prime}\right)\right|^{2}
\end{array}
$$

(b) Radiation polarized parallel to magnetic field

$$
\begin{array}{lll}
\left|f_{B}\left(\theta^{\prime}, \phi\right)\right|^{2} & 0 & \left|f_{B}\left(\theta^{\prime}+\pi / 2, \phi\right)\right|^{2} \\
0 & \left|f_{B}\left(-\theta, \pi / 2-\phi^{\prime}\right)\right|^{2} & 0 \\
\left|f_{B}\left(\theta^{\prime}, \phi+\pi / 2\right)\right|^{2} & 0 & \left|f_{B}\left(\theta^{\prime}+\pi / 2, \phi+\pi / 2\right)\right|^{2} \\
0 & \left|f_{B}\left(-\theta,-\phi^{\prime}\right)\right|^{2} & 0
\end{array}
$$

(c) Radiation with left circular polarization

$$
\begin{array}{lll}
\left|f_{+}\left(\frac{\pi}{2}-\theta^{\prime}, \frac{\pi}{2}-\phi^{\prime}\right)\right|^{2} & 0 & \left|f_{-}(\theta+\pi / 2, \phi)\right|^{2} \\
0 & \left|f_{-}(\theta+\pi / 2, \phi+\pi / 2)\right|^{2} & 0 \\
\left|f_{+}\left(\frac{\pi}{2}-\theta^{\prime},-\phi^{\prime}\right)\right|^{2} & 0 & \left|f_{+}\left(-\theta^{\prime}, \frac{\pi}{2}-\phi^{\prime}\right)\right|^{\prime}
\end{array}
$$

(d) Radiation with right circular polarization

$$
\begin{array}{lll}
\left|f_{-}\left(\frac{\pi}{2}-\theta^{\prime}, \frac{\pi}{2}-\phi^{\prime}\right)\right|^{2} & 0 & \left|f_{+}(\theta+\pi / 2, \phi)\right|^{2} \\
0 & \left|f_{+}(\theta+\pi / 2, \phi+\pi / 2)\right|^{2} & 0 \\
\left|f_{-}\left(\frac{\pi}{2}-\theta^{\prime},-\phi^{\prime}\right)\right|^{2} & 0 & \left|f_{-}\left(-\theta^{\prime},-\theta^{\prime}, \frac{\pi}{2}-\phi^{\prime}\right)\right|^{2}
\end{array}
$$


then

$$
|\widehat{\mathbf{q}} \cdot \mathbf{F}(\hat{\mathbf{X}}, \hat{\mathbf{Y}} ; \theta, \phi)|^{2}=\cos ^{2} \eta\left|f_{T}(\theta, \phi)\right|^{2}+\sin ^{2} \eta\left|f_{T}^{\prime}(\theta, \phi)\right|^{2}+\frac{1}{2} \sin 2 \eta\left(f_{T}^{*} f_{T}^{\prime}+f_{T} f_{T}^{\prime *}\right),
$$

where

$$
f_{T}^{*} f_{T}^{\prime}+f_{T} f_{T}^{\prime *}=\frac{w N}{2}\{-6+3[\cos 2 \theta+\cos 2 \phi+\cos 2 \theta \cos 2 \phi]+5 \sin 2 \theta \sin 2 \phi-\sqrt{3}[\sin 2 \theta+\sin 2 \phi-\sin (2 \theta+2 \phi)]\} .
$$

If $\mathbf{B}=0$, both $2 \theta$ and $2 \phi$ are $\pi / 3$ and the expression on the right of (105) vanishes. Unlike the other cases already considered, in the absence of stress the term in $w$ may persist; this was implicit in the work of Ref. 8 .

The polarization vectors for left and right circular polarization are, respectively,

$$
\hat{\mathbf{q}}_{L}=(\hat{\mathbf{X}}+i \hat{\mathbf{Y}}) / \sqrt{2} \text { and } \hat{\mathbf{q}}_{R}=(\hat{\mathbf{X}}-i \hat{\mathbf{Y}}) / \sqrt{2} \text {. }
$$

Once more the tabulation of intensities is convenient in terms of amplitudes

$$
f_{-}(\theta, \phi)=\frac{1}{\sqrt{2}}\left[f_{T}(\theta, \phi)-i f_{T}^{\prime}(\theta, \phi)\right]
$$

and

$$
f_{+}(\theta, \phi)=\frac{1}{\sqrt{2}}\left[f_{T}(\theta, \phi)+i f_{T}^{\prime}(\theta, \phi)\right] .
$$

The intensities for circular polarization are then

$$
\begin{aligned}
\left|\widehat{\mathbf{q}}_{L} \cdot \mathbf{F}(\hat{\mathbf{X}}, \hat{\mathbf{Y}} ; \theta, \phi)\right|^{2} & =\left|\hat{\mathbf{q}}_{R} \cdot \mathbf{F}(\hat{\mathbf{X}},-\hat{\mathbf{Y}} ; \theta, \phi)\right|^{2} \\
& =\left|f_{+}\right|^{2} \\
& =\frac{1}{2}\left\{\left|f_{T}\right|^{2}+\left|f_{T}^{\prime}\right|^{2}+i\left[f_{T}^{*} f_{T}^{\prime}-f_{T} f_{T}^{\prime *}\right]\right\}
\end{aligned}
$$

and

$$
\begin{aligned}
\left|\hat{\mathbf{q}}_{R} \cdot \mathbf{F}(\hat{\mathbf{X}}, \hat{\mathbf{Y}} ; \theta, \phi)\right|^{2} & =\left|\hat{\mathbf{q}}_{L} \cdot \mathbf{F}(\hat{\mathbf{X}},-\hat{\mathbf{Y}} ; \theta, \phi)\right|^{2} \\
& =\left|f_{-}\right|^{2} \\
& =\frac{1}{2}\left\{\left|f_{T}\right|^{2}+\left|f_{T}^{\prime}\right|^{2}-i\left[f_{T}^{*} f_{T}^{\prime}-f_{T} f_{T}^{\prime *}\right]\right\},
\end{aligned}
$$

where

$$
\begin{array}{r}
f_{T}^{*} f_{T}^{\prime}-f_{T} f_{T}^{\prime *}=\frac{i N}{4}[\cos (2 \phi-\pi / 3)-\cos (2 \theta-\pi / 3)]+\frac{i N u}{8}\{3[\cos (2 \theta-\pi / 3)-\cos (2 \phi-\pi / 3)]-\sqrt{3} \sin (2 \theta-2 \phi)\} \\
-\frac{i N v}{16}\{2+3[\cos 2 \theta+\cos 2 \phi-\sin 2 \theta \sin 2 \phi] \\
-5 \cos 2 \theta \cos 2 \phi-\sqrt{3}[\sin 2 \theta+\sin 2 \phi-\sin (2 \theta+2 \phi)]\} .
\end{array}
$$

A succinct tabulation of the intensities for individual transitions for selected polarizations is given in Table VI.

\section{Case 4: stress parallel to [111], magnetic field parallel to [110]}

\section{Energies and wave functions}

As for case 3 , the crystal symmetry elements common to the stress and the magnetic field are $E, \bar{E}, \sigma$, and $\bar{\sigma}$, so the symmetry group is again $C_{s}$. The magnetic field is the same as for case 3, so the Zeeman Hamiltonians are identical to those of Eqs. (74) and (75). The stress Hamiltonians are constructed from the general forms, Eqs. (4) and (6), by use of the strain components $\varepsilon_{x x}=\varepsilon_{y y}$ $=\varepsilon_{z z}=\frac{1}{3} T\left(s_{11}+2 s_{12}\right)$ and $\varepsilon_{x y}=\varepsilon_{y z}=\varepsilon_{z x}=\frac{1}{6} T s_{44}$. For the $\Gamma_{6}$ and $\Gamma_{7}$ states, the form is unaltered from that of case 3 , so in total for these states there is no change in the interactions, and the energies, eigenfunctions, and symmetries are identical to those of Eqs. (76) - (79).

For the $\Gamma_{8}$ states the stress Hamiltonian is 


$$
\begin{aligned}
H_{p}^{(8)}= & {\left[a^{\prime}\left(s_{11}+2 s_{12}\right)-\frac{5}{6 \sqrt{3}} d^{\prime} s_{44}\right] T I } \\
& +\frac{d^{\prime} T}{6 \sqrt{3}} s_{44}\left(J_{x}+J_{y}+J_{z}\right)^{2} .
\end{aligned}
$$

The first stage of the diagonalization procedure is identical to that used for case 3 . The intermediate functions are those of Eqs. (80) and (81). Using these functions the full piezo-Zeeman Hamiltonian becomes that indicated in Eq. (82) and has the form given in Eq. (16), where the values of the parameters are now as follows:

$$
\begin{aligned}
& h_{0}=a^{\prime}\left(s_{11}+2 s_{12}\right) T+B^{2}\left[q_{1}+\frac{5}{4}\left(q_{2}+q_{3}\right)\right] \\
& h_{1}=\mu_{B} B\left(\frac{3}{2} g_{1}^{\prime}+3 g_{2}^{\prime}\right) \\
& h_{2}=\frac{d^{\prime} T}{4 \sqrt{3}} s_{44}-\left(q_{2}+\frac{1}{4} q_{3}\right) B^{2} \\
& h_{3}=(1+i 2 \sqrt{2}) \frac{d^{\prime} T}{12} s_{44}+\frac{3 \sqrt{3}}{8} \mu_{B} g_{2}^{\prime} B+\frac{\sqrt{3}}{4} q_{3} B^{2} \\
& h_{3}^{\prime}=(1+i 2 \sqrt{2}) \frac{d^{\prime} T}{12} s_{44}-\frac{3 \sqrt{3}}{8} \mu_{B} g_{2}^{\prime} B+\frac{\sqrt{3}}{4} q_{3} B^{2} \\
& h_{4}=\mu_{B} B\left(\frac{1}{2} g_{1}^{\prime}+\frac{5}{4} g_{2}^{\prime}\right) .
\end{aligned}
$$

The energy eigenvalues and symmetry identifications are those of Eqs. (89a)-(89d), with Eqs. (22)-(25) supplying the coefficients of the intermediate functions in full expansions of the eigenfunctions.

\section{Intensities}

The three orthogonal directions useful for the expression of the transition amplitudes for the present case are obtained in elementary fashion, and are

$$
\begin{aligned}
& \hat{\mathbf{X}}=(\widehat{\mathbf{x}}+\hat{\mathbf{y}}+\widehat{\mathbf{z}}) / \sqrt{3}, \\
& \hat{\mathbf{Y}}=(\widehat{\mathbf{x}}+\hat{\mathbf{y}}-2 \widehat{\mathbf{z}}) / \sqrt{6}, \\
& \hat{\mathbf{Z}}=(\hat{\mathbf{y}}-\widehat{\mathbf{x}}) / \sqrt{2} .
\end{aligned}
$$

The display of some transition amplitudes is assisted by defining angle $\beta$ through the relation

$$
\sqrt{3} e^{i \beta}=1+i \sqrt{2} .
$$

a. $\Gamma_{8} \rightarrow \Gamma_{6}$ transitions. The eigenfunctions derived from the $\Gamma_{6}\left(\bar{T}_{d}\right)$ states were formed by rotating the original functions, and those derived from the $\Gamma_{8}\left(\bar{T}_{d}\right)$ states used a rotation and a further unitary transformation. If all of these transformations are applied in the appropriate manner to the matrix of Eq. (7), the amplitude matrix is obtained for the transitions in the presence of the stress and magnetic field as
$-D_{0}\left\{\begin{array}{c}\sqrt{3}(\hat{\mathbf{X}}+i \hat{\mathbf{Y}}) \cos \theta e^{i \beta} \\ +(\hat{\mathbf{X}}-i \hat{\mathbf{Y}}) \sin \theta e^{-i(\alpha+\beta)} \\ -2 \hat{\mathbf{Z}} \sin \theta e^{-i \alpha}\end{array}\right.$

$$
\begin{gathered}
(\hat{\mathbf{X}}-i \hat{\mathbf{Y}}) \cos \theta e^{-i \beta} \\
-\sqrt{3}(\hat{\mathbf{X}}+i \hat{\mathbf{Y}}) \sin \theta e^{i(\alpha+\beta)} \\
-2 \hat{\mathbf{Z}} \cos \theta
\end{gathered}
$$

$$
\left.\begin{array}{c}
-2 i \widehat{\mathbf{Z}} \sin \theta^{\prime} e^{i \alpha^{\prime}} \\
i \sqrt{3}(\widehat{\mathbf{X}}-i \widehat{\mathbf{Y}}) \cos \theta^{\prime} e^{-i \beta} \\
-i(\widehat{\mathbf{X}}+i \widehat{\mathbf{Y}}) \sin \theta^{\prime} e^{i\left(\alpha^{\prime}+\beta\right)}
\end{array}\right)
$$

Intensities derived from these amplitudes are listed in Table VII.

b. $\quad \Gamma_{8} \rightarrow \Gamma_{7}$ transitions. The procedure leading from Eq. (7) to amplitude matrix (94) is now applied to Eq. (8) and produces the following matrix of relative transition amplitudes.

$$
D_{0}^{\prime}\left(\begin{array}{cccc}
i \sqrt{3} \sin \theta e^{-i \alpha} \hat{\mathbf{Z}} & \sqrt{2}\left[\cos \theta^{\prime}-\sin \theta^{\prime} e^{\left.-i \gamma^{\prime}\right]} \hat{\mathbf{Y}}\right. & i \sqrt{3} \cos \theta \hat{\mathbf{Z}} & {\left[\sin \theta^{\prime} e^{i \alpha^{\prime}}-\sqrt{3} \cos \theta^{\prime} e^{-2 i \beta}\right] \hat{\mathbf{X}}} \\
+i \cos \theta \hat{\mathbf{Z}} & -\left[\cos \theta^{\prime}+\sqrt{3} \sin \theta^{\prime} e^{-i \delta^{\prime}}\right] \hat{\mathbf{X}} & -i \sin \theta e^{i \alpha} \hat{\mathbf{Z}} & -\sqrt{2}\left[\sin \theta^{\prime} e^{i \alpha^{\prime}}+\cos \theta^{\prime} e^{-i \beta}\right] \hat{\mathbf{Y}} \\
i\left[\sqrt{3} \cos \theta e^{2 i \beta}+\sin \theta e^{-i \alpha}\right] \hat{\mathbf{X}} & \sin \theta^{\prime} e^{-i \alpha^{\prime}} \hat{\mathbf{Z}} & i\left[\cos \theta-\sqrt{3} \sin \theta e^{i \delta}\right] \hat{\mathbf{X}} & \cos \theta^{\prime} \hat{\mathbf{Z}} \\
+i \sqrt{2}\left[\cos \theta e^{i \beta}-\sin \theta e^{-i \alpha}\right] \hat{\mathbf{Y}} & +\sqrt{3} \cos \theta^{\prime} \hat{\mathbf{Z}} & -i \sqrt{2}\left[\cos \theta+\sin \theta e^{i \gamma}\right] \hat{\mathbf{Y}} & -\sqrt{3} \sin \theta^{\prime} e^{i \alpha^{\prime}} \hat{\mathbf{Z}}
\end{array}\right) .
$$

Symbols used in the matrix are $\gamma=\alpha+\beta, \delta=\alpha+2 \beta, \gamma^{\prime}=\alpha^{\prime}+\beta$, and $\delta^{\prime}=\alpha^{\prime}+2 \beta$. Intensities arising from these amplitudes for selected polarizations are included in Table VII.

c. $\Gamma_{8} \rightarrow \Gamma_{8}$ transitions. The method of constructing the unitary transformation needed to diagonalize the Hamiltonian has been described already, and must be applied to both bra and ket manifolds. The consequent transformation of Eq. (9) then yields the appropriate amplitudes. For the cases previously considered, the parameters $\alpha$ and $\alpha^{\prime}$ defined in Eq. (23) have not made an explicit appearance as symbols in transition amplitudes because they had simple numeric values. For the present case such symbols must be retained; however, for the bra states they will be replaced by $\eta$ and $\eta^{\prime}$, respectively, together with the replacement of $\theta$ and $\theta^{\prime}$ by $\phi$ and $\phi^{\prime}$, respectively.

As in Eqs. (32), (60), and (99), the amplitude matrix separates naturally for polarization components parallel to and orthogonal to the magnetic field. The amplitudes for components parallel to the magnetic field are 
$\stackrel{\Im}{\Xi}$
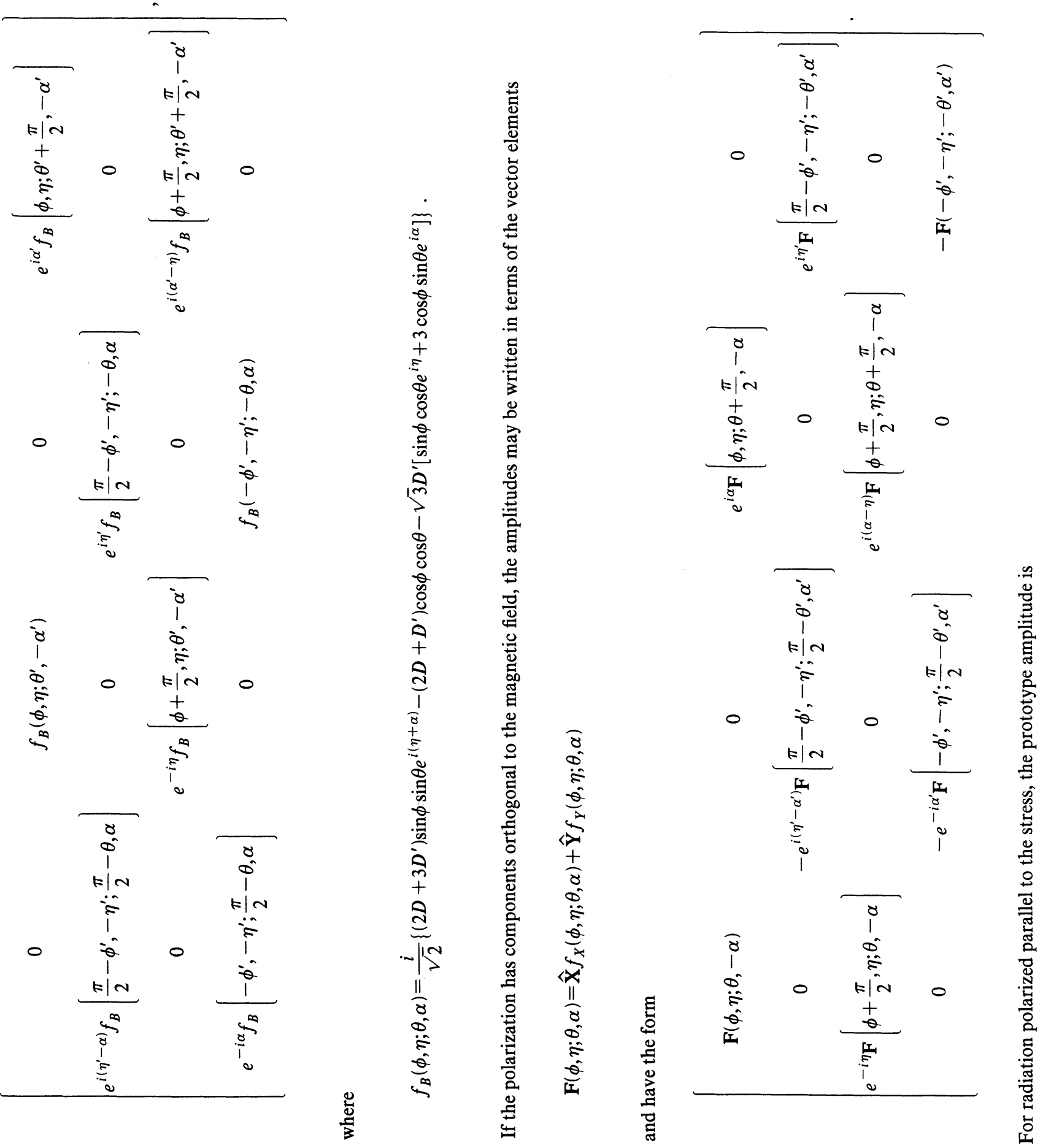
TABLE VII. $\Gamma_{8} \rightarrow \Gamma_{6}$ and $\Gamma_{8} \rightarrow \Gamma_{7}$ transitions. Relative intensities for $\mathbf{B} \|[\overline{1} 10]$ with $\mathbf{T} \|[111]$. Angles $2 \theta$ and $2 \theta^{\prime}$ are defined in terms of field, stress, and coupling parameters in Eqs. (24) and (25), respectively, but the parameters $h_{1}-h_{4}$ entering those definitions are those defined in Eqs. (114)-(118). The angle $\beta$ is defined in Eq. (122). The functions indicated in the column headed $\bar{T}_{d}$ are rotated so that the axis of quantization is parallel to the magnetic field, becoming the functions listed in the column headed $\bar{C}_{s}$.

\begin{tabular}{clllll}
\hline \hline $\bar{T}_{d}$ & $\bar{C}_{s}$ & $\Gamma_{3}$ & $\Gamma_{4}$ & $\Gamma_{3^{\prime}}$ & $\Gamma_{4^{\prime}}$ \\
\hline
\end{tabular}

A. Linear polarization

$\begin{array}{lll}\Gamma_{6}\left(\frac{1}{2}\right) & \Gamma_{3} & 1+\frac{1}{2} \cos 2 \theta \\ & & +\frac{\sqrt{3}}{2} \sin 2 \theta \cos (\alpha+2 \beta) \\ \Gamma_{6}\left(-\frac{1}{2}\right) & \Gamma_{4} & 0 \\ \Gamma_{7}\left(\frac{1}{2}\right) & \Gamma_{4} & 0 \\ \Gamma_{7}\left(-\frac{1}{2}\right) & \Gamma_{3} & 1+\frac{1}{2} \cos 2 \theta \\ & & +\frac{\sqrt{3}}{2} \sin 2 \theta \cos (\alpha+2 \beta)\end{array}$

(a) Radiation polarized parallel to stress

0

$$
\begin{aligned}
& 1-\frac{1}{2} \cos 2 \theta \\
& -\frac{\sqrt{3}}{2} \sin 2 \theta \cos (\alpha+2 \beta)
\end{aligned}
$$

$1-\frac{1}{2} \cos 2 \theta^{\prime}$

0

$+\frac{\sqrt{3}}{2} \sin 2 \theta^{\prime} \cos \left(\alpha^{\prime}+2 \beta\right)$

$1-\frac{1}{2} \cos 2 \theta^{\prime}$

0

$+\frac{\sqrt{3}}{2} \sin 2 \theta^{\prime} \cos \left(\alpha^{\prime}+2 \beta\right)$

0

$$
\begin{aligned}
& 1-\frac{1}{2} \cos 2 \theta \\
& -\frac{\sqrt{3}}{2} \sin 2 \theta \cos (\alpha+2 \beta)
\end{aligned}
$$

$1+\frac{1}{2} \cos 2 \theta^{\prime}$

$-\frac{\sqrt{3}}{2} \sin 2 \theta^{\prime} \cos \left(\alpha^{\prime}+2 \beta\right)$

$1+\frac{1}{2} \cos 2 \theta^{\prime}$

$-\frac{\sqrt{3}}{2} \sin 2 \theta^{\prime} \cos \left(\alpha^{\prime}+2 \beta\right)$

0

(b) Radiation polarized parallel to the magnetic field

$$
\begin{array}{lll}
\Gamma_{6}\left(\frac{1}{2}\right) & \Gamma_{3} & 0 \\
\Gamma_{6}\left(-\frac{1}{2}\right) & \Gamma_{4} & 1-\cos 2 \theta \\
\Gamma_{7}\left(\frac{1}{2}\right) & \Gamma_{4} & 1-\frac{1}{2} \cos 2 \theta \\
& & +\frac{\sqrt{3}}{2} \sin 2 \theta \cos \alpha
\end{array}
$$$$
1+\cos 2 \theta^{\prime} \quad 0
$$$$
0
$$$$
0
$$$$
1+\cos 2 \theta
$$$$
1+\frac{1}{2} \cos 2 \theta
$$$$
-\frac{\sqrt{3}}{2} \sin 2 \theta \cos \alpha
$$

$1+\frac{1}{2} \cos 2 \theta^{\prime}$

$$
0
$$$$
+\frac{\sqrt{3}}{2} \sin 2 \theta^{\prime} \cos \alpha^{\prime}
$$

$$
\begin{aligned}
& 1-\cos 2 \theta^{\prime} \\
& 0 \\
& 0 \\
& 1-\frac{1}{2} \cos 2 \theta^{\prime} \\
& -\frac{\sqrt{3}}{2} \sin 2 \theta^{\prime} \cos \alpha^{\prime}
\end{aligned}
$$

B. Circular polarization

(a) Radiation with left circular polarization

$$
\begin{array}{lll}
\Gamma_{6}\left(\frac{1}{2}\right) & \Gamma_{3} & \frac{1}{2}(1-\cos 2 \theta) \\
\Gamma_{6}\left(-\frac{1}{2}\right) & \Gamma_{4} & 0 \\
\Gamma_{7}\left(\frac{1}{2}\right) & \Gamma_{4} & 0 \\
& & \\
\Gamma_{7}\left(-\frac{1}{2}\right) & \Gamma_{3} & \frac{3}{2}+\frac{3}{4} \cos 2 \theta \\
& & -(3 \sqrt{3} / 4) \sin 2 \theta \cos \alpha
\end{array}
$$

0

$\frac{3}{2}\left(1-\cos 2 \theta^{\prime}\right)$

$\frac{1}{2}+\frac{1}{4} \cos 2 \theta^{\prime}$

$+\frac{\sqrt{3}}{4} \sin 2 \theta^{\prime} \cos \alpha^{\prime}$

0

(b) Radiation with right circular polarization

$$
\begin{array}{lll}
\Gamma_{6}\left(\frac{1}{2}\right) & \Gamma_{3} & \frac{3}{2}(1+\cos 2 \theta) \\
\Gamma_{6}\left(-\frac{1}{2}\right) & \Gamma_{4} & 0 \\
\Gamma_{7}\left(\frac{1}{2}\right) & \Gamma_{4} & 0 \\
\Gamma_{7}\left(-\frac{1}{2}\right) & \Gamma_{3} & \frac{1}{2}-\frac{1}{4} \cos 2 \theta \\
& & +\frac{\sqrt{3}}{4} \sin 2 \theta \cos \alpha
\end{array}
$$

$-\frac{\sqrt{3}}{4} \sin 2 \theta \cos \alpha$

$$
\begin{array}{ll}
\frac{1}{2}(1+\cos 2 \theta) & 0 \\
0 & \frac{3}{2}\left(1+\cos 2 \theta^{\prime}\right) \\
0 & \frac{1}{2}-\frac{1}{4} \cos 2 \theta^{\prime} \\
& -\frac{\sqrt{3}}{4} \sin 2 \theta^{\prime} \cos \alpha^{\prime}
\end{array}
$$$$
\frac{3}{2}-\frac{3}{4} \cos 2 \theta
$$$$
0
$$$$
+(3 \sqrt{3} / 4) \sin 2 \theta \cos \alpha
$$

0

0

$\frac{1}{2}\left(1-\cos 2 \theta^{\prime}\right)$

$\frac{3}{2}+\frac{3}{4} \cos 2 \theta^{\prime}$

$+(3 \sqrt{3} / 4) \sin 2 \theta^{\prime} \cos \alpha^{\prime}$
$0 \quad \frac{1}{2}+\frac{1}{4} \cos 2 \theta \quad 0$ 
$f_{X}(\phi, \eta ; \theta, \alpha)=\hat{\mathbf{X}} \cdot \mathbf{F}(\phi, \eta ; \theta, \alpha)$

$$
\begin{aligned}
&=\frac{1}{2 \sqrt{3}}\{ {\left[\sqrt{3}\left(D+D^{\prime}\right)+i \sqrt{6} D^{\prime}\right] \cos \phi \cos \theta-\left[D+5 D^{\prime}+i \sqrt{2}\left(2 D+D^{\prime}\right)\right] \cos \phi \sin \theta e^{i \alpha} } \\
&\left.-\left[D-3 D^{\prime}-i \sqrt{2}\left(2 D+3 D^{\prime}\right)\right] \sin \phi \cos \theta e^{i \eta}-\left[\sqrt{3}\left(D+D^{\prime}\right)-i 3 \sqrt{6} D^{\prime}\right] \sin \phi \sin \theta e^{i(\alpha+\eta)}\right\} .
\end{aligned}
$$

For left circular polarization the prototype amplitude is

$f_{+}(\phi, \eta ; \theta, \alpha)=\frac{1}{\sqrt{2}}(\widehat{\mathbf{X}}+i \widehat{\mathbf{Y}}) \cdot \mathbf{F}(\phi, \eta ; \theta, \alpha)$

$$
=\frac{1}{2}\left(\frac{1}{\sqrt{2}}-i\right)\left\{D \cos \phi \cos \theta+\frac{1}{\sqrt{3}}\left(D-4 D^{\prime}\right) \cos \phi \sin \theta e^{i \alpha}-\sqrt{3} D \sin \phi \cos \theta e^{i \eta}-\left(D+4 D^{\prime}\right) \sin \phi \sin \theta e^{i(\alpha+\eta)}\right\}
$$

and for right circular polarization the prototype amplitude is

$$
\begin{aligned}
& f_{-}(\phi, \eta ; \theta, \alpha)= \frac{1}{\sqrt{2}}(\hat{\mathbf{X}}-i \hat{\mathbf{Y}}) \cdot \mathbf{F}(\phi, \eta ; \theta, \alpha) \\
&=\frac{1}{2}\left(\frac{1}{\sqrt{2}}+i\right)\left\{\left(D+2 D^{\prime}\right) \cos \phi\left[\cos \theta-\sqrt{3} \sin \theta e^{i \alpha}\right]\right. \\
& \\
&\left.\quad+\frac{1}{\sqrt{3}}\left(D+6 D^{\prime}\right) \sin \phi \cos \theta e^{i \eta}+\left(2 D^{\prime}-D\right) \sin \phi \sin \theta e^{i(\alpha+\eta)}\right\} .
\end{aligned}
$$

Should it be needed, $f_{Y}(\phi, \eta ; \theta, \alpha)$, the amplitude for linear polarization orthogonal to both stress and field, can be obtained by subtraction using Eqs. (129) and (130), but for the sake of economy of space it has been omitted here.

The intensities for the separate transitions arising from these amplitudes are much too cumbersome to display even in tabular form, so only the prototype intensities are listed here. Intensities for the individual transitions may be deduced from the prototypes by the use of elementary trigonometrical relationships with reference to the angles shown as arguments of the elements of matrices (125) and (127):

$$
\begin{aligned}
& \left|f_{B}(\phi, \eta ; \theta, \alpha)\right|^{2}=\frac{N}{2}\{1+\cos 2 \phi \cos 2 \theta-\sin 2 \phi \sin 2 \theta \cos (\eta+\alpha)\} \\
& +\frac{N u}{16}\{6 \cos 2 \phi-6 \cos 2 \theta-15 \cos 2 \phi \cos 2 \theta-\sqrt{3}[(2-\cos 2 \theta) \sin 2 \phi \cos \eta+(2+\cos 2 \phi) \sin 2 \theta \cos \alpha] \\
& \left.+\left[\frac{9}{2} \cos (\eta-\alpha)+\frac{17}{2} \cos (\eta+\alpha)\right] \sin 2 \theta \sin 2 \phi\right\} \\
& +\frac{N v}{4}\{\cos 2 \phi+\cos 2 \theta+\sqrt{3}[(1-2 \cos 2 \theta) \sin 2 \phi \cos \eta-(1+2 \cos 2 \phi) \sin 2 \theta \cos \alpha]\} \\
& +N w\{\sqrt{3}[(2+\cos 2 \phi) \sin 2 \theta \sin \alpha+(2-\cos 2 \theta) \sin 2 \phi \sin \eta]-2 \sin 2 \theta \sin 2 \phi \sin (\eta+\alpha)\}, \\
& \left|f_{X}(\phi, \eta ; \theta, \alpha)\right|^{2}=\frac{N}{12}\left\{\frac{3}{2}(\cos 2 \theta+\cos 2 \phi)-\sqrt{3}\left[\left(\frac{1}{4} \cos \alpha+\frac{1}{\sqrt{2}} \sin \alpha\right) \sin 2 \theta \cos 2 \phi+\left[\frac{1}{4} \cos \eta+\frac{1}{\sqrt{2}} \sin \eta\right] \sin 2 \phi \cos 2 \theta\right]\right. \\
& \left.-\frac{3}{4} \cos 2 \theta \cos 2 \phi-\left[\frac{3}{8} \cos (\eta-\alpha)+\frac{7}{8} \cos (\eta+\alpha)+\frac{1}{\sqrt{2}} \sin (\eta+\alpha)\right] \sin 2 \phi \sin 2 \theta\right\} \\
& +\frac{N u}{48}\left\{\frac{9}{2} \cos 2 \theta \cos 2 \phi+\left[\frac{15}{4} \cos (\eta-\alpha)+\frac{7}{4} \cos (\eta+\alpha)+\sqrt{2} \sin (\eta+\alpha)\right] \sin 2 \phi \sin 2 \theta\right. \\
& -3(\cos 2 \theta+\cos 2 \phi)+\sqrt{3}\left[\sin 2 \theta(2+\cos 2 \phi)\left(\frac{1}{2} \cos \alpha+\sqrt{2} \sin \alpha\right)\right. \\
& \left.\left.+\sin 2 \phi(2+\cos 2 \theta)\left(\frac{1}{2} \cos \eta+\sqrt{2} \sin \eta\right)\right]\right\} \\
& +\frac{N v}{24} \sqrt{\frac{3}{2}}\{\sin 2 \phi \cos 2 \theta(\sin \eta-2 \sqrt{2} \cos \eta)+2 \sqrt{2} \sin (\eta-\alpha)-\sin 2 \theta \cos 2 \phi(\sin \alpha-2 \sqrt{2} \cos \alpha)\} \\
& -\frac{N w}{6 \sqrt{2}}\{12-6(\cos 2 \theta+\cos 2 \phi-\cos 2 \theta \cos 2 \phi)-[3 \cos (\eta-\alpha)+7 \cos (\eta+\alpha)+4 \sqrt{2} \sin (\eta+\alpha)] \sin 2 \phi \sin 2 \theta \\
& +\sqrt{2} \sin 2 \theta(1-\cos 2 \phi)(\sqrt{6} \cos \alpha+8 \sin \alpha)+\sqrt{2} \sin 2 \phi(1-\cos 2 \theta)(\sqrt{6} \cos \eta+8 \sin \eta)\},
\end{aligned}
$$




$$
\begin{aligned}
\left|f_{-}(\phi, \eta ; \theta, \alpha)\right|^{2}= & \frac{N}{16}\left\{2-v+\left\{1-\frac{9 u}{4}-2 v\right] \cos 2 \phi-\left[1-\frac{3 u}{4}+v\right] \cos 2 \theta-\frac{1}{2}\left[1-\frac{3 u}{4}-5 v\right] \cos 2 \phi \cos 2 \theta\right\} \\
& +\frac{N}{8 \sqrt{3}}(1+\cos 2 \theta)\left[\left(1+\frac{u}{4}-3 v\right] \cos \eta+16 w \sin \eta\right] \sin 2 \phi \\
& -\frac{N}{8 \sqrt{3}}(1-\cos 2 \phi)\left[\left[1-\frac{19 u}{4}-v\right] \cos \alpha-32 w \sin \alpha\right] \sin 2 \theta \\
& +\frac{N \sqrt{3}}{8}(1-\cos 2 \theta)\left[\left[1-\frac{7 u}{4}+v\right] \cos \eta-16 w \sin \eta\right] \sin 2 \phi \\
& -\frac{N \sqrt{3}}{8}(1+\cos 2 \phi)\left[1-\frac{3 u}{4}-v\right] \cos \alpha \sin 2 \theta \\
& -\left\{\frac{N}{8}\left[\left(1-\frac{7 u}{4}+v\right] \cos (\eta+\alpha)+\left[1+\frac{u}{4}-3 v\right) \cos (\eta-\alpha)\right]-4 w N \cos \eta \sin \alpha\right\} \sin 2 \theta \sin 2 \phi
\end{aligned}
$$

$$
\begin{aligned}
\left|f_{+}(\phi, \eta ; \theta, \alpha)\right|^{2}= & \frac{N}{16}\left\{2+v+\left[1-\frac{9 u}{4}+2 v\right] \cos 2 \theta-\left[1-\frac{3 u}{4}-v\right] \cos 2 \phi-\frac{1}{2}\left[1-\frac{3 u}{4}+5 v\right] \cos 2 \phi \cos 2 \theta\right\} \\
& +\frac{N}{8 \sqrt{3}}(1+\cos 2 \phi]\left[\left(1+\frac{u}{4}+3 v\right] \cos \alpha-16 w \sin \alpha\right] \sin 2 \theta \\
& -\frac{N}{8 \sqrt{3}}(1-\cos 2 \theta)\left[\left[1-\frac{19 u}{4}+v\right] \cos \eta+32 w \sin \eta\right] \sin 2 \phi \\
& +\frac{N \sqrt{3}}{8}(1-\cos 2 \phi)\left[\left[1-\frac{7 u}{4}-v\right] \cos \alpha+16 w \sin \alpha\right] \sin 2 \theta \\
& -\frac{N \sqrt{3}}{8}(1+\cos 2 \theta)\left[1-\frac{3 u}{4}+v\right] \cos \eta \sin 2 \phi \\
& -\left\{\frac{N}{8}\left[\left(1-\frac{7 u}{4}-v\right] \cos (\eta+\alpha)+\left[1+\frac{u}{4}+3 v\right] \cos (\eta-\alpha)\right]+4 w N \cos \alpha \sin \eta\right\} \sin 2 \theta \sin 2 \phi
\end{aligned}
$$

If it is desired to calculate the intensities for polarizations other than those indicated, the amplitudes are constructed by the use of Eqs. (125)-(130), and the intensities deduced in terms of the intensity parameters $u, v$, and $w$ by application of the defining relations (33) $-(35)$.

\section{THE SIGNIFICANCE OF $w$}

For transitions between two manifolds of $\Gamma_{8}$ states, group theory permits two, and only two, independent complex matrix elements, designated here and in other works as $D$ and $D^{\prime}$. Apart from the overall normalization factor $N$, in previous studies these have given rise to just two intensity parameters, designated here and elsewhere as $u$ and $v$. Why then was it necessary to introduce a third intensity parameter designated above as $w$ ? Indeed, how is it possible?

Let $D$ and $D^{\prime}$ be expressed in terms of their amplitudes and phases:

$$
D=|D| e^{i \lambda}, \quad D^{\prime}=\left|D^{\prime}\right| e^{i \lambda^{\prime}} .
$$

Set

$$
r=\left|D^{\prime}\right| /|D| \text {. }
$$

The defining relations for $u, v$, and $w$, Eqs. (33), (34), (35), and (38), then become

$$
\begin{aligned}
& |D|^{2}\left[1+r^{2}+2 r \cos \left(\lambda-\lambda^{\prime}\right)\right]=\frac{1}{4} N(1-u), \\
& |D|^{2}\left[1+r^{2}-2 r \cos \left(\lambda-\lambda^{\prime}\right)\right]=\frac{1}{4} N(1+2 v), \\
& |D|^{2}\left[1+9 r^{2}+6 r \cos \left(\lambda-\lambda^{\prime}\right)\right]=\frac{1}{4} N(1-2 v), \\
& |D|^{2} r \sin \left(\lambda-\lambda^{\prime}\right)=N w .
\end{aligned}
$$

It is clear from these relationships that measurements of $u$ and $v$ can yield the value of $\cos \left(\lambda-\lambda^{\prime}\right)$ and thereby the magnitude of the relative phase difference between $D$ and $D^{\prime}$, but only a measurement of $w$ can yield its sign. Thus $w$ is not an independent parameter of the theory, and therefore its use does not conflict with the requirements of group theory, but it does contain new information, namely the sign of the relative phase. No measure- 
ments of $w$ have been reported for any system. With known values of $u$ and $v$, Eqs. (137) -(140) can be used to compute its magnitude, and this may assist in the reduction of data to yield the value of $w$ including its sign. The foregoing theory provides a range of combinations of directions of field, stress, and polarization which should offer convenience in the determination of $w$, and with it potentially useful new information.

\section{CONCLUSION}

Since the procedures described here employ an exact diagonalization of the Hamiltonians, the validity of the theory is limited only by the validity of the Hamiltonians employed. It is unlikely that quadratic terms would need to be introduced into the stress Hamiltonians, and quadratic terms have been explicitly included in the Zeeman Hamiltonians. Thus the only point of possible vulnerability of the predictions is interaction that could arise between adjacent manifolds of states. Application of some of these results have already been made and reported elsewhere. ${ }^{12}$ For boron, gallium, and thallium impurities in germanium, the fit of the experimental data to the energy expressions were described as "excellent," although some excited states gave some indication of interaction between neighboring manifolds. Consistency in the $g$ factors deduced from different orientations of the field was obtained. It is therefore reasonable to conclude that the results presented here strengthen the application of piezoZeeman spectroscopy in the task of characterization of impurity states in semiconductors.

\section{APPENDIX}

Following details given in Ref. 10, basis functions which can generate the angular momentum matrices and the representations of $\bar{T}_{d}$ that are relevant to the present work are as follows:

$$
\phi_{1 / 2}^{(6)}=f_{0}(r)|\alpha\rangle, \quad \phi_{-1 / 2}^{(6)}=f_{0}(r)|\beta\rangle,
$$

where $f_{0}(r)$ is a normalized $s$ function, $|\alpha\rangle$ and $|\beta\rangle$ are the spin- $\frac{1}{2}$ spinors, and the functions $\phi_{1 / 2}^{(6)}$ and $\phi_{-1 / 2}^{(6)}$ belong to $\Gamma_{6}\left(\bar{T}_{d}\right)$. Functions transforming as $\Gamma_{7}\left(\bar{T}_{d}\right)$ can be generated from normalized $p$ functions, $X=x f(r)$, $Y=y f(r)$, and $Z=z f(r)$, together with $|\alpha\rangle$ and $|\beta\rangle$. These are

$$
\begin{aligned}
& \phi_{1 / 2}^{(7)}=\frac{1}{\sqrt{3}}[(X+i Y)|\beta\rangle+Z|\alpha\rangle], \\
& \phi_{-1 / 2}^{(7)}=\frac{1}{\sqrt{3}}[(X-i Y)|\alpha\rangle-Z|\beta\rangle] .
\end{aligned}
$$

The $\Gamma_{6}$ and $\Gamma_{7}$ functions each generate the following forms for the $j=\frac{1}{2}$ angular momentum matrices:

$$
\begin{aligned}
& j_{x}=\frac{1}{2}\left[\begin{array}{ll}
0 & 1 \\
1 & 0
\end{array}\right], j_{y}=\frac{1}{2}\left[\begin{array}{cc}
0 & -i \\
i & 0
\end{array}\right], \\
& j_{z}=\frac{1}{2}\left[\begin{array}{ll}
1 & 0 \\
0 & 1
\end{array}\right] .
\end{aligned}
$$

Functions which generate $\Gamma_{8}\left(\bar{T}_{d}\right)$ are

$$
\begin{aligned}
& \phi_{3 / 2}^{(8)}=(1 / \sqrt{2})(X+i Y)|\alpha\rangle, \\
& \phi_{1 / 2}^{(8)}=(i / \sqrt{6})[(X+i Y)|\beta\rangle-2 Z|\alpha\rangle], \\
& \phi_{-1 / 2}^{(8)}=(1 / \sqrt{6})[(X-i Y)|\alpha\rangle+2 Z|\beta\rangle], \\
& \phi_{-3 / 2}^{(8)}=(i / \sqrt{2})(X-i Y)|\beta\rangle .
\end{aligned}
$$

The $J=\frac{3}{2}$ angular momentum matrices generated by these functions are

$$
\begin{aligned}
& J_{x}=\frac{i}{2}\left[\begin{array}{cccc}
0 & \sqrt{3} & 0 & 0 \\
-\sqrt{3} & 0 & 2 & 0 \\
0 & -2 & 0 & \sqrt{3} \\
0 & 0 & -\sqrt{3} & 0
\end{array}\right], \\
& J_{y}=\frac{1}{2}\left[\begin{array}{cccc}
0 & \sqrt{3} & 0 & 0 \\
\sqrt{3} & 0 & 2 & 0 \\
0 & 2 & 0 & \sqrt{3} \\
0 & 0 & \sqrt{3} & 0
\end{array}\right],
\end{aligned}
$$

and

$$
J_{z}=\frac{1}{2}\left[\begin{array}{cccc}
3 & 0 & 0 & 0 \\
0 & 1 & 0 & 0 \\
0 & 0 & -1 & 0 \\
0 & 0 & 0 & -3
\end{array}\right]
$$

There are other basis functions which transform the same way as the ones shown here, and these have been listed in Appendix D of Ref. 10. The functions $\psi_{\mu}^{(6)}, \psi_{\mu}^{(7)}$, and $\psi_{\mu}^{(8)}$ used in the main text in the present work are taken to be in the most general form, that is, they are assumed to be linear combinations of functions drawn from the available sets of functions, and thus they have greater generality than the functions shown above.
${ }^{1}$ A. K. Ramdas and S. Rodriguez, Rep. Prog. Phys. 44, 1297 (1981); C. Jagannath, Z. W. Grabowski, and A. K. Ramdas, Phys. Rev. B 23, 2082 (1981); A. D. Martin et al., Phys. Lett. 99A, 391 (1983); J. W. Cross et al., Phys. Rev. B 28, 6953 (1983); E. H. Salib, P. Fisher, and P. E. Simmonds, ibid. 32, 2424 (1985); R. A. Lewis, P. Fisher, and N. A. McLean, in IOP Conf. Proc. No. 95, edited by B. Monemar, p. 95 (Institute of Physics and Physical Society, London, 1989).
${ }^{2}$ C. A. Freeth, P. Fisher, and P. E. Simmonds, Solid State Commun. 60, 175 (1986); C. A. Freeth, P. Fisher, and R. E. M. Vickers, in Proceedings of the 18th International Conference on the Physics of Semiconductors, Stockholm, 1986, edited by O. Engstrom (World Scientific, Singapore, 1987), Vol. 2, p. 841; P. Fisher, C. A. Freeth, and R. E. M. Vickers, Physica 146B, 80 (1987).

${ }^{3}$ A. Baldereschi and N. O. Lipari, Phys. Rev. B 8, 2697 (1973); 
9, 1525 (1974); N. O. Lipari and A. Baldereschi, Solid State Commun. 25, 665 (1978); N. Binggeli and A. Baldereschi, ibid. 66, 323 (1988); V. Fiorentini and A. Baldereschi, ibid. 69, 953 (1989); M. Said, M. A. Kanehisa, and M. Jouanne, in Proceedings of the 18th International Conference on the Physics of Semiconductors, Stockholm, 1986, edited by O. Engstrom (World Scientific, Singapore, 1987), Vol. 2, p. 983.

${ }^{4}$ R. Buckzo, Nuovo Cimento 9D, 669 (1987); G. L. Bir and G. E. Pikus, Symmetry and Strain-Induced Effects in Semiconductors (Wiley, New York, 1974); J. Broeckx and J. Vennik, Phys. Rev. B 35, 6165 (1987)

${ }^{5}$ J. Broeckx, Phys. Rev. B 43, 9643 (1991-II); W. O. G. Schmidt, E. Bangert, and G. Landwehr, J. Phys. Condens. Matter 3, 6789 (1991); P. J. Lin-Chung, and R. F. Wallis, J. Phys. Chem. Solids 30, 1453 (1969).

${ }^{6}$ Y. Chen, B. Gil, and H. Mathieu, Ann. Phys. 12, 109 (1987).

${ }^{7}$ H. R. Chandrasekhar, P. Fisher, A. K. Ramdas, and S. Rodriguez, Phys. Rev. B 8, 3836 (1973); K. J. Duff, P. Fisher, and
N. R. Butler, Aust. J. Phys. 33, 73 (1980).

${ }^{8}$ S. Rodriguez, P. Fisher, and F. Barra, Phys. Rev. B 5, 2219 (1972).

${ }^{9}$ E. Kartheuser and S. Rodriguez, Phys. Rev. B 8, 1556 (1973); E. H. Salib and K. J. Duff, ibid. 33, 1275 (1986).

${ }^{10}$ A. K. Bhattercharjee and S. Rodriguez, Phys. Rev. B 6, 3836 (1972).

${ }^{11}$ B. R. Judd and W. A. Runciman, Proc. R. Soc. London, Ser. A 352, 91 (1976).

${ }^{12}$ K. J. Duff et al., in Proceedings of the 19th International Conference on the Physics of Semiconductors, edited by W. Zawadski (Institute of Physics, Polish Academy of Science, Warsaw, 1988), p. 1273; K. J. Duff et al., in Proceedings of the 20th International Conference on the Physics of Semiconductors, Thessaloniki, 1990, edited by E. M. Anastassakis and J. D. Joannopoulos (World Scientific, Singapore, 1990), p. 642.

${ }^{13}$ M. Villeret and S. Rodriguez, Nuovo Cimento D 13, 529 (1991). 